\title{
Shape morphing and topology optimization of fluid channels by explicit boundary tracking
}

Zhou, Mingdong; Lian, Haojie; Sigmund, Ole; Aage, Niels

Published in:

International Journal for Numerical Methods in Fluids

Link to article, DOI:

10.1002/fld. 4667

Publication date:

2018

Document Version

Peer reviewed version

Link back to DTU Orbit

Citation (APA):

Zhou, M., Lian, H., Sigmund, O., \& Aage, N. (2018). Shape morphing and topology optimization of fluid channels by explicit boundary tracking. International Journal for Numerical Methods in Fluids, 88(6), 296-313.

https://doi.org/10.1002/fld.4667

\section{General rights}

Copyright and moral rights for the publications made accessible in the public portal are retained by the authors and/or other copyright owners and it is a condition of accessing publications that users recognise and abide by the legal requirements associated with these rights.

- Users may download and print one copy of any publication from the public portal for the purpose of private study or research.

- You may not further distribute the material or use it for any profit-making activity or commercial gain

- You may freely distribute the URL identifying the publication in the public portal 
DOI: $\mathrm{xxx} / \mathrm{xxxx}$

\title{
RESEARCH ARTICLE
}

\section{Shape morphing and topology optimization of fluid channels by explicit boundary tracking}

\author{
Mingdong Zhou*1 | Haojie Lian² | Ole Sigmund ${ }^{3}$ | Niels Aage
}

\author{
${ }^{1}$ State Key Laboratory of Mechanical \\ System and Vibration, Shanghai Key \\ Laboratory of Digital Manufacture for \\ Thin-walled Structures, Shanghai Jiao Tong \\ University, P. R. China. \\ ${ }^{2}$ College of Mining Engineering, Taiyuan \\ University of Technology, P. R. China. \\ ${ }^{3}$ Section of Solid Mechanics, Department of \\ Mechanical Engineering, Technical \\ University of Denmark, Denmark
}

\section{Correspondence}

*Mingdong Zhou, Rm. A801, Building of Mechanical Engineering, No.800 Dongchuan Road, Minhang District, Shanghai, P. R. China.Email: mdzhou@sjtu.edu.cn

\begin{abstract}
An integrated shape morphing and topology optimization approach based on the Deformable Simplicial Complex (DSC) methodology is developed to address Stokes and Navier-Stokes flow problems. The optimized geometry is interpreted by a set of piecewise linear curves embedded in a well-formed triangular mesh, resulting in a physically well-defined interface between fluid and impermeable regions. The shape evolution is realized by deforming the curves while maintaining a high-quality mesh through adaption of the mesh near the structural boundary, rather than performing a global remeshing. Topological changes are allowed through hole merging or splitting of islands. The finite element discretization used, provides smooth and stable optimized boundaries for simple energy dissipation objectives. However, for more advanced problems boundary oscillations are observed due to conflicts between objective function and minimum length-scale imposed by the meshing algorithm. A surface regularization scheme is introduced to circumvent this issue, which is specifically tailored for the DSC approach. In contrast to other filter-based regularization techniques, the scheme does not introduce additional control variables and at the same time it is based on rigorous sensitivity analysis. Several numerical examples are presented to demonstrate the applicability of the approach.
\end{abstract}

\section{KEYWORDS:}

Shape optimization; Topology optimization; Stokes flow; Navier-Stokes flow; Explicit boundary tracking; Deformable simplicial complex

\section{1 | INTRODUCTION}

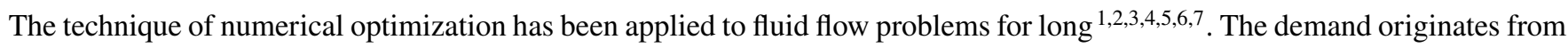
the aerospace industry, e.g. airfoil design, where a small shape modification leads to drag reduction and results in tremendous saving in energy and cost. Now it has become popular in a broad range of other application fields, e.g. from lab-on-a-chip devices to general fluid control systems such as pipes, fluid mixers, switches and valves.

Central to the shape optimization is the geometric parametrization. For a designer, the most intuitive way to perform shape optimization is still by modifying the parameters of a Computer Aided Geometric Design (CAGD) based representation, such as B-splines ${ }^{819}$, NURBS ${ }^{10}$ and T-splines ${ }^{11}$ which facilitates a CAD to CAD model workflow. Physical simulation in this way can be decoupled from the geometric representation and hidden from designers. However, since a particular parameterization implies a reduced design space for optimization, a clever decision must be made in order to find the right balance between the 
parameterization, the optimal solution space and the computational time, which in practice may not be an easy task ${ }^{12}$. Another often used approach for shape optimization is to directly deform a computational grid ${ }^{713}$, e.g. a finite element mesh, for which the grid not only represents the geometry but also is used for simulation. Given a certain level of discretization, one will have the most design freedom for shape optimization when all boundary nodes are assigned as design variables. However if the topological connectivity of a mesh is fixed, only a limited shape deformation can be achieved before the mesh gets severely distorted and the physical simulation aborts. A truly optimal shape can only be realized if the mesh is allowed to be adaptive and of high quality. Recently, the Deformable Simplicial Complex (DSC) method ${ }^{[14}$ was developed to support large geometric deformation by explicitly moving the boundary nodes whilst maintaining a well-formed mesh through local mesh adaption. This method utilizes a simplicial complex (a triangular mesh in 2D and a tetrahedron mesh in 3D) to represent the geometry as well as to support finite element analysis. The elements are annotated as either solid or void while the target designable boundary is sandwiched in-between. As a result, this method can ensure a high quality mesh during large shape deformation by altering only the degenerated or badly shaped element near the interface during the shape evolution, rather than performing a global remeshing. The alteration is implemented in an efficient way based on a set of mesh-quality rules and local mesh operations such as smoothing, edge split, edge collapse and face removal. It possesses the advantages of using explicit boundary representation for yielding functionally optimized geometries with maximum design freedom (w.r.t. a given discretization). Previously, the DSC method has been applied to shape and topology optimization of structural compliance minimization problems $\frac{15[16}{\text { as well }}$ as stress minimization 17$] 18$. In this paper, it is further developed and applied to optimization of Stokes and Navier-Stokes flow problems, with support of hole splitting and merging. Although the proposed approach yields stable results for simple energy dissipation objectives, a new regularization scheme is proposed to control feature sizes and to ensure smoothed optimized designs for more advanced problems. The regularization scheme, which is particularly developed for the DSC framework, is different from existing methods $\frac{13 \mid 12}{12}$ that employ a designable parameter model and a physical geometry. Here, only one set of boundary nodes is considered that represents both the design variables and the actual geometry. The proposed regularization scheme is found effective in obtaining convergent results for certain Navier-Stokes flow problems (c.f. Section 6.3), when the considered design objective is particularly sensitive to local geometric changes.

The above discussion are mainly addressing design from a shape optimization perspective. However, enormous successes have been made on topology optimization for fluid flow, fluid-structure interaction and fluid based heat transfer problems. For density-based topology optimization approaches, interested readers are referred to some recent work 19201212223124125] as well as the references therein. In addition, the level set based topology optimization approach ${ }^{26}$ is another powerful tool for shape and topology optimization which has been applied to fluid problems 2728 29/30131. Common for most density and levelset based topology optimization approaches based on fixed background meshes is that resulting boundaries between fluid and solid are described by jagged edges and/or elements taking intermediate fluid properties through density interpolations or ersatz material properties. Although in general providing reliable results, such blurry boundary conditions may in some cases have troubles in capturing refined flow and boundary effects and hence, a number of level set based papers have considered explicit conformal structural meshing for accurate evaluation of the signed-distance function ${ }^{32}$ and for mechanical analysis $33 / 34$. As

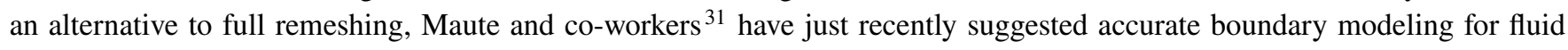
problems using a CutFEM approach. The DSC approach for topology and shape optimization of fluid problems suggested in this paper represents: an alternative way of imposing accurate boundary conditions; possibility for topological changes through merging and splitting of holes and including procedures for ensuring well-formed boundary conforming meshes. Ignoring its ability to perform topological changes, the method closely follows and yields similar results to classical shape optimization papers from the literature. However, together with the CutFEM approach ${ }^{31}$, the presented methodology is so far unique in its ability to perform simultaneous shape and topology optimization of fluid problems.

The remainder of the paper is organized as follows. In Section 2, the Deformable Simplicial Complex methodology is briefly reviewed. Then, the details of the shape optimization problem and sensitivity analysis are given in Section 3 and 4, respectively. The optimization procedure is summarized in Section 5. Several benchmark examples are presented in Section 6. Besides, the new regularization scheme is also introduced and discussed. Conclusions are given afterwards.

\section{2 | DEFORMABLE SIMPLICIAL COMPLEX}

The Deformable Simplicial Complex (DSC) ${ }^{14}$ is a method for deforming an explicit surface via mesh operations. Fig. 1 a) illustrates its application to 2D fluid flow problems. A domain of interest is first discretized using a triangular mesh, of which each 
element belongs to either the solid or the fluid phase whilst the interface is sandwiched in-between. The interface is interpreted by a series of piecewise linear curves. Shape deformation is then enabled by moving the nodes on the interface from an old position $\mathbf{w}^{\text {old }}$ to a new position $\mathbf{w}^{\text {new }}$ along their normal directions. During a deformation process, local mesh adaption is performed near the interface to fix any degenerated elements. By doing so, a well-formed mesh is guaranteed during the shape updating process and thus a large deformation can be realized. This method has been previously applied to fluid simulation ${ }^{35}$ as well as shapetopology optimization for structural compliance minimization problems ${ }^{\frac{15}{156}}$. For the shape optimization, the design variable for each boundary node $\mathbf{w}_{i}$ is the length of deformation $\tau_{i}$ in its outward normal direction:

$$
\mathbf{w}_{i}^{\text {new }}=\mathbf{w}_{i}^{\text {old }}+\tau_{i} \mathbf{n}_{i} .
$$

Note that during the DSC-based shape optimization, initial disconnected features, such as holes (for elastic problems) or islands (for fluid problems), can merge or disappear which corresponds to topological change. The opposite case of introducing new holes or islands is less likely to happen and must be aided through topological derivatives as e.g. done for elasticity in 15 .

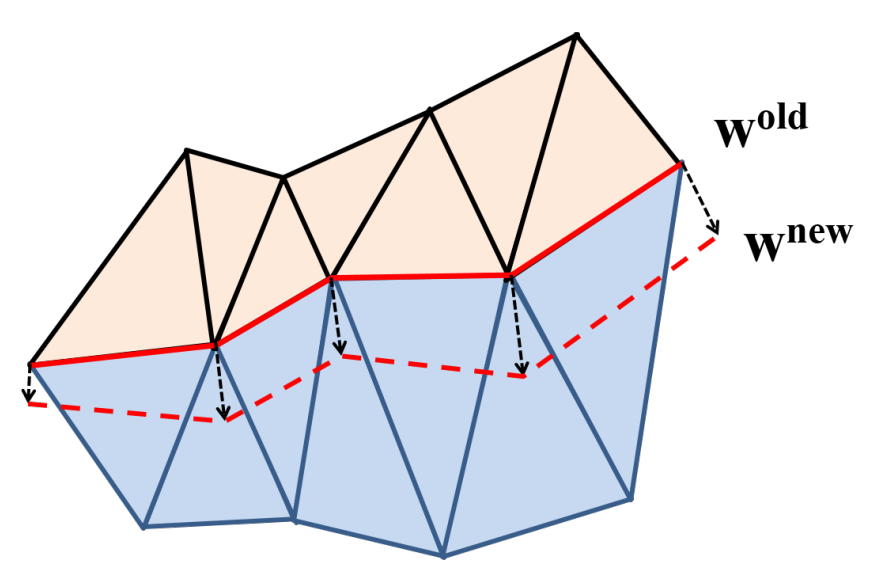

(a) DSC boundary update

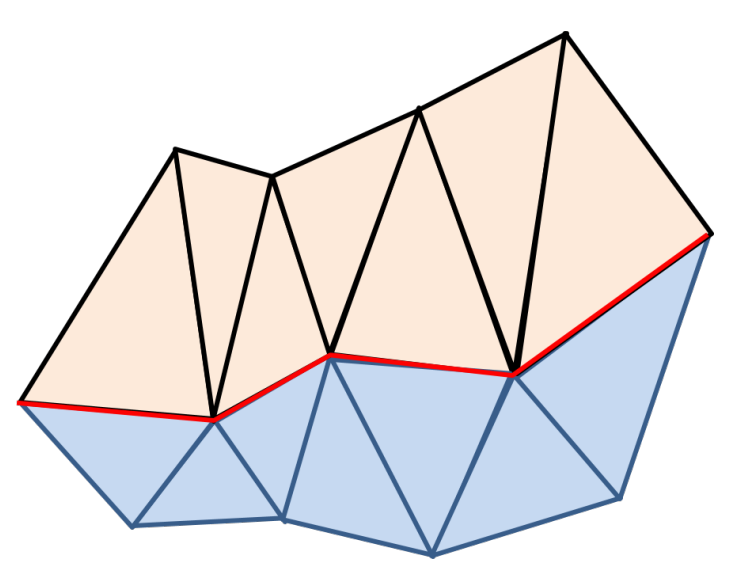

(b) reformed mesh

FIGURE 1 Explicit boundary representation and geometry update by the DSC method: the designable boundary (red color) is sandwiched between the fluid (blue color) and the solid (brown color) phase. (a) the boundary nodes move from $\mathbf{w}^{\text {old }}$ to $\mathbf{w}^{\text {new }}$ along their normal directions; (b) the reformed mesh

\section{3 | OPTIMIZATION PROBLEM}

\section{1 | Governing equations}

This paper assumes steady-state incompressible flow with low to moderate Reynolds numbers. The governing equations are given as:

$$
\begin{array}{rlr}
\text { Momentum equation : } & \rho \frac{\partial u_{i}}{\partial x_{j}} u_{j}-\frac{\partial \sigma_{i j}}{\partial x_{j}}-b_{i}=0, & \text { in } \Omega, \\
\text { Incompressibility : } & \frac{\partial u_{i}}{\partial x_{i}}=0, & \text { in } \Omega, \\
\text { Boundary condition : } & \sigma_{i j} n_{j}=t_{i}, & \text { on } \Gamma_{\sigma}, \\
: & u_{i}=u_{i}^{*}, & \text { on } \Gamma_{u},
\end{array}
$$


where $\rho$ denotes the density of the fluid, $u$ is the velocity, $b$ is the body force, $t$ is the traction, $u^{*}$ is a prescribed velocity and subscripts $i, j$ define the spatial directions, respectively. The stress tensor $\sigma_{i j}$ is defined as:

$$
\sigma_{i j}=\mu\left(\frac{\partial u_{i}}{\partial x_{j}}+\frac{\partial u_{j}}{\partial x_{i}}\right)-p \delta_{i j}
$$

where $p$ denotes pressure, $\mu$ dynamic viscosity and $\delta_{i j}$ stands for Kronecker's delta defined as

$$
\delta_{i j}= \begin{cases}1, & \text { if } i=j \\ 0, & \text { else }\end{cases}
$$

The Galerkin weak forms of the above steady-state incompressible Navier-Stokes equations (1) are written as:

$$
\begin{aligned}
R_{1}\left(u_{i}, p ; v_{i}\right)= & \int_{\Omega} v_{i}\left(\rho \frac{\partial u_{i}}{\partial x_{j}} u_{j}-\frac{\partial}{\partial x_{j}}\left(\mu\left(\frac{\partial u_{i}}{\partial x_{j}}+\frac{\partial u_{j}}{\partial x_{i}}\right)-p \delta_{i j}\right)-b_{i}\right) d \Omega \\
= & \int_{\Omega} v_{i} \rho \frac{\partial u_{i}}{\partial x_{j}} u_{j} d \Omega+\int_{\Omega}\left(\frac{\partial v_{i}}{\partial x_{j}}+\frac{\partial v_{j}}{\partial x_{i}}\right) \mu\left(\frac{\partial u_{i}}{\partial x_{j}}+\frac{\partial u_{j}}{\partial x_{i}}\right) d \Omega- \\
& \int_{\Omega}\left(\frac{\partial v_{i}}{\partial x_{j}}+\frac{\partial v_{j}}{\partial x_{i}}\right) p \delta_{i j} d \Omega-\int_{\Gamma} v_{i} n_{j} \sigma_{i j} d \Gamma-\int_{\Omega} v_{i} b_{i} d \Omega=0, \\
R_{2}\left(u_{i}, p ; q\right)= & \int_{\Omega} q \frac{\partial u_{i}}{\partial x_{i}} d \Omega=0,
\end{aligned}
$$

where $R$ is the residual, $v_{i}$ and $q$ are the admissible velocity and pressure test functions, respectively. The non-dimensional Reynolds number $R e$ is used to characterize the fluid behavior:

$$
R e=\frac{\rho \bar{u} L}{\mu},
$$

where $\bar{u}$ and $L$ are the reference velocity and length, respectively. Generally, a high or a low Re corresponds to a turbulent or a laminar flow, respectively. Note that by setting $\rho=0.0$ in the above, the governing equations for Stokes flow are recovered. In such a flow problem, the velocity is small while the fluid viscosity is large. Hence, the nonlinear convection term $\frac{\partial u_{i}}{\partial x_{j}} u_{j}$ is neglected and the resulting linear equation can be solved very efficiently.

\section{2 | Finite element formulation}

The DSC discretization provides a well-formed and conforming mesh for finite element analysis. For the fluid simulation in the current work, the order of the finite element is chosen to be quadratic in velocities and piecewise constant for the pressure. A schematic illustration for such an element as well as the designable nodes $\square^{1}$ is shown in Fig. 2 Such a velocity-pressure formulation is proven to be stable and adequate to simulate the fluid flow of small to moderate $R e$ without relying on extra stabilization 36 . After discretization, the finite element formulation is given as:

$$
\begin{gathered}
{\left[\begin{array}{cc}
\mathbf{C}(\mathbf{u})+\mathbf{K}_{11}, \mathbf{K}_{12} \\
\mathbf{K}_{21} & \mathbf{0}
\end{array}\right]\left[\begin{array}{l}
\mathbf{u} \\
\mathbf{p}
\end{array}\right]=\left[\begin{array}{l}
\mathbf{F} \\
\mathbf{0}
\end{array}\right],} \\
\mathbf{C}(\mathbf{u})=\sum_{e} \mathbf{C}^{e}(\mathbf{u}), \mathbf{K}_{12}=\sum_{e} \mathbf{K}_{12}^{e}, \mathbf{K}_{21}=\sum_{e} \mathbf{K}_{21}^{e}, \mathbf{F}=\sum_{e} \mathbf{F}^{e},
\end{gathered}
$$

where $\mathbf{C}(\mathbf{u})$ is the convection matrix, $\mathbf{K}_{11}$ is the viscosity matrix, $\mathbf{K}_{12}$ and $\mathbf{K}_{21}$ are the coupling matrix, $\mathbf{u}$ and $\mathbf{p}$ are the nodal velocity and pressure vectors, respectively, and $\mathbf{F}$ is the load vector. The corresponding element-wise contribution is denoted

\footnotetext{
${ }^{1} \mathrm{~A}$ corner node is designable only if it becomes the boundary node.
} 
with a subscript $e$, for which the individual terms are integrated as:

$$
\begin{aligned}
\mathbf{C}^{e}(\mathbf{u}) & =\rho \int_{\Omega_{e}} \mathbf{N}_{u}^{\mathrm{T}} \mathbf{D}_{u}^{\mathrm{T}} \mathbf{T}_{c} \mathbf{B}_{u} d \Omega, \\
\mathbf{K}_{11}^{e} & =\mu \int_{\Omega_{e}} \mathbf{B}_{u}^{\mathrm{T}} \mathbf{T}_{k} \mathbf{B}_{u} d \Omega, \\
\mathbf{K}_{12}^{e} & =\int_{\Omega_{e}} \mathbf{B}_{u}^{\mathrm{T}} \mathbf{T}_{g} \mathbf{N}_{p} d \Omega, \\
\mathbf{K}_{21}^{e} & =\left(\mathbf{K}_{12}^{e}\right)^{\mathrm{T}}, \\
\mathbf{F}^{e} & =\int_{\Omega_{e}} \mathbf{N}_{u}^{\mathrm{T}} \mathbf{t} \mathbf{d} \mathbf{\Gamma},
\end{aligned}
$$

where

$$
\begin{aligned}
& \mathbf{N}_{u}=\left[\begin{array}{ccccccc}
N_{u 1}, & 0, & N_{u 2}, & 0, & \ldots, & N_{u 6}, & 0 \\
0, & N_{u 1}, & 0, & N_{u 2}, & \ldots, & 0, & N_{u 6}
\end{array}\right], \\
& \mathbf{N}_{p}=[1] \text {, } \\
& \mathbf{D}_{u}=\left[\begin{array}{cc}
\mathbf{N}_{u} \mathbf{u}_{e}, & 0 \\
0, & \mathbf{N}_{u} \mathbf{u}_{e},
\end{array}\right], \\
& \mathbf{B}_{u}=\left[\begin{array}{ccccccc}
\frac{\partial N_{u 1}}{\partial x}, & 0, & \frac{\partial N_{u 2}}{\partial x}, & 0, & \ldots, & \frac{\partial N_{u 6}}{\partial x}, & 0 \\
0, & \frac{\partial N_{u 1}}{\partial x}, & 0, & \frac{\partial N_{u 2}}{\partial x}, & \ldots, & 0, & \frac{\partial N_{u 6}}{\partial x} \\
\frac{\partial N_{u 1}}{\partial y}, & 0, & \frac{\partial N_{u 2}}{\partial y}, & 0, & \ldots, & \frac{\partial N_{u 6}}{\partial y}, & 0 \\
0, & \frac{\partial N_{u 1}}{\partial y}, & 0, & \frac{\partial N_{u 2}}{\partial y}, & \ldots, & 0, & \frac{\partial N_{u 6}}{\partial y}
\end{array}\right],
\end{aligned}
$$

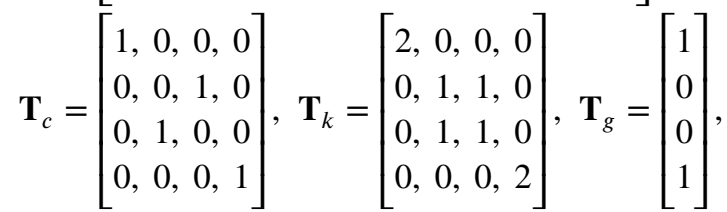

where $\mathbf{N}$ is the shape function matrix, subscripts $u$ and $p$ denote the associated definition of velocity and pressure, respectively. Other matrices in Eqs. (96]10) are defined for matrix multiplication purposes. The non-linear Navier-Stokes equation (7) is solved by a hybrid solver based on a fixed-value iteration approach and a Newton-type solver 37 .

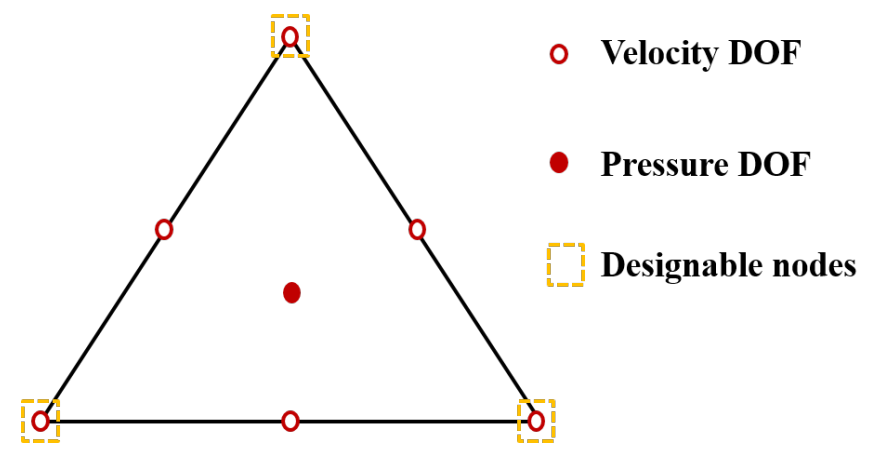

FIGURE 2 Degree of freedom (DOF) for velocity and pressure and designable nodes in a triangular finite element. 


\section{4 | SENSITIVITY ANALYSIS}

Provided that the design variable of the DSC is the domain variation vector $\tau$ of the boundary nodes, the shape derivative for a general objective functional $\Phi(\mathbf{u}(\boldsymbol{\tau}), \mathbf{p}(\boldsymbol{\tau}), \boldsymbol{\tau})$ is obtained by:

$$
\frac{d \Phi}{d \boldsymbol{\tau}}=\frac{\partial \Phi}{\partial \boldsymbol{\tau}}+\lambda^{\mathrm{T}} \frac{\partial \mathbf{R}}{\partial \boldsymbol{\tau}},
$$

where $\mathbf{R}$ is the vector form of the residual after discretization:

$$
\mathbf{R}=\left[\begin{array}{cc}
\mathbf{C}(\mathbf{u})+\mathbf{K}_{11}, & \mathbf{K}_{12} \\
\mathbf{K}_{21} & \mathbf{0}
\end{array}\right]\left[\begin{array}{l}
\mathbf{u} \\
\mathbf{p}
\end{array}\right]-\left[\begin{array}{l}
\mathbf{F} \\
\mathbf{0}
\end{array}\right] .
$$

The adjoint vector $\lambda$, containing the adjoint variables for both velocity and pressure, is obtained by solving the following linear equation:

$$
\left(\frac{\partial \mathbf{R}}{\partial \mathbf{v}}\right)^{\mathrm{T}} \lambda=\frac{\partial \Phi}{\partial \mathbf{v}},
$$

where $\mathbf{v}=[\mathbf{u}, \mathbf{p}]^{\mathrm{T}}$. The element-wise contributions are defined as:

$$
\frac{\partial \mathbf{R}}{\partial \mathbf{v}}=\sum_{e}\left(\frac{\partial \mathbf{R}}{\partial \mathbf{v}}\right)^{e}, \frac{\partial \mathbf{R}}{\partial \tau}=\sum_{e}\left(\frac{\partial \mathbf{R}}{\partial \tau}\right)^{e},
$$

and the corresponding terms are given as:

$$
\begin{gathered}
\left(\frac{\partial \mathbf{R}}{\partial \mathbf{v}}\right)^{e}=\left[\begin{array}{cc}
\mathbf{S}_{1}^{e}+\mathbf{S}_{2}^{e}+\mathbf{K}_{11}^{e}, \mathbf{K}_{12}^{e} \\
\mathbf{K}_{21}^{e} & \mathbf{0}
\end{array}\right], \\
\mathbf{S}_{1}^{e}=\rho \int_{\Omega_{e}} \mathbf{N}_{u}^{\mathrm{T}} \mathbf{D}_{u}^{\mathrm{T}} \mathbf{T}_{c} \mathbf{B}_{u} d \Omega, \\
\mathbf{S}_{2}^{e}=\rho \int_{\Omega_{e}} \mathbf{N}_{u}^{\mathrm{T}} \mathbf{G}_{u} \mathbf{N}_{u} d \Omega, \\
\mathbf{G}_{u}=\left[\begin{array}{ll}
\sum_{j=1}^{12}\left(\mathbf{B}_{u}\right)_{1 j} \mathbf{u}_{j}, & \sum_{j=1}^{12}\left(\mathbf{B}_{u}\right)_{2 j} \mathbf{u}_{j} \\
\sum_{j=1}^{12}\left(\mathbf{B}_{u}\right)_{3 j} \mathbf{u}_{j}, & \sum_{j=1}^{12}\left(\mathbf{B}_{u}\right)_{4 j} \mathbf{u}_{j}
\end{array}\right],
\end{gathered}
$$

and

$$
\begin{gathered}
\mathbf{B}_{u}=\left[\begin{array}{ccccccc}
\frac{\partial N_{u 1}}{\partial x}, & 0, & \frac{\partial N_{u 2}}{\partial x}, & 0, & \ldots, & \frac{\partial N_{u 6}}{\partial x}, & 0 \\
0, & \frac{\partial N_{u 1}}{\partial x}, & 0, & \frac{\partial N_{u 2}}{\partial x}, & \ldots, & 0, & \frac{\partial N_{u 6}}{\partial x} \\
\frac{\partial N_{u 1}}{\partial y}, & 0, & \frac{\partial N_{u 2}}{\partial y}, & 0, & \ldots, & \frac{\partial N_{u 6}}{\partial y}, & 0 \\
0, & \frac{\partial N_{u 1}}{\partial y}, & 0, & \frac{\partial N_{u 2}}{\partial y}, & \ldots, & 0, & \frac{\partial N_{u 6}}{\partial y}
\end{array}\right], \\
\left(\frac{\partial \mathbf{R}}{\partial \tau}\right)^{e}=\frac{\partial}{\partial \tau}\left[\begin{array}{ccc}
\mathbf{C}^{e}(\mathbf{u})+\mathbf{K}_{11}^{e}, & \mathbf{K}_{12}^{e} \\
\mathbf{K}_{21}^{e} & \mathbf{0}
\end{array}\right] \cdot\left[\begin{array}{c}
\mathbf{u}^{e} \\
\mathbf{p}^{e}
\end{array}\right],
\end{gathered}
$$




$$
\begin{aligned}
\frac{\partial}{\partial \boldsymbol{\tau}} \mathbf{C}^{e}(\mathbf{u})= & \rho \int_{\Omega_{p}} \mathbf{N}_{u}^{\mathrm{T}} \mathbf{D}_{u}^{\mathrm{T}} \mathbf{T}_{c} \frac{\partial \mathbf{B}_{u}}{\partial \tau} \mathbf{J} d \Omega+\rho \int_{\Omega_{p}} \mathbf{N}_{u}^{\mathrm{T}} \mathbf{D}_{u}^{\mathrm{T}} \mathbf{T}_{c} \mathbf{B}_{u} \frac{\partial \mathbf{J}}{\partial \boldsymbol{\tau}} d \Omega, \\
\frac{\partial}{\partial \boldsymbol{\tau}} \mathbf{K}_{11}^{e}= & \mu \int_{\Omega_{p}}\left(\frac{\partial \mathbf{B}_{u}}{\partial \boldsymbol{\tau}}\right)^{\mathrm{T}} \mathbf{T}_{k} \mathbf{B}_{u} \mathbf{J} d \Omega+\mu \int_{\Omega_{p}} \mathbf{B}_{u}^{\mathrm{T}} \mathbf{T}_{k} \frac{\partial \mathbf{B}_{u}}{\partial \tau} \mathbf{J} d \Omega \\
& +\mu \int_{\Omega_{p}} \mathbf{B}_{u}^{\mathrm{T}} \mathbf{T}_{k} \mathbf{B}_{u} \frac{\partial \mathbf{J}}{\partial \boldsymbol{\tau}} d \Omega, \\
\frac{\partial}{\partial \boldsymbol{\tau}} \mathbf{K}_{12}^{e}= & \int_{\Omega_{p}}\left(\frac{\partial \mathbf{B}_{u}}{\partial \boldsymbol{\tau}}\right)^{\mathrm{T}} \mathbf{T}_{g} \mathbf{N}_{p} \mathbf{J} d \Omega+\int_{\Omega_{p}} \mathbf{B}_{u}^{\mathrm{T}} \mathbf{T}_{g} \mathbf{N}_{p} \frac{\partial \mathbf{J}}{\partial \tau} d \Omega, \\
\frac{\partial}{\partial \tau} \mathbf{K}_{21}^{e}= & \frac{\partial}{\partial \boldsymbol{\tau}}\left(\mathbf{K}_{12}^{e}\right)^{\mathrm{T}},
\end{aligned}
$$

where $\Omega_{p}$ is the parent element in parametric coordinates and $\mathbf{J}$ is the Jacobian matrix.

\section{5 | OPTIMIZATION PROCEDURE}

The solution procedure for the presented optimization solution is summarized as:

Step 1 Establish a DSC geometric FE model and setup optimization parameters.

Step 2 Solve the primary problem Eq. (7).

Step 3 Solve the adjoint problem Eq. 13.

Step 4 Evaluate the shape gradients using Eq. (11).

Step 5 Solve the optimization problem using the Method of Moving Asymptotes 38 .

Step 6 Update the position of all designable nodes and perform local mesh adaption to guarantee mesh quality.

Step 7 Repeat steps 2-7 until convergence.

Details regarding Step 6 can be found in reference 15 .

\section{6 | NUMERICAL EXAMPLES}

\section{1 | Football design problem under Stokes flow}

The first example revisits a benchmark shape optimization problem of designing an optimized profile of minimum drag under Stokes flow ${ }^{39}$. Fig. 3 a) illustrates the problem setup, where an infinite flow with a density value $2^{2} \rho=0.0$ enters a rectangular design domain from the left inlet with a uniform velocity of $u_{x}=1, u_{y}=0$ and exits from the right edge with the same velocity. The top and bottom boundaries are non-penetrating slip walls imitating periodicity while the designable obstacle boundary is assumed to be no-slip. The optimization problem is to minimize the viscous dissipated energy subject to a volume constraint on

\footnotetext{
${ }^{2}$ The units are non-dimensional for all the examples in this paper.
} 
TABLE 1 Statistics of the football design example in Fig. 3

\begin{tabular}{c|c|c}
\hline Optimization Cases & Viscous energy dissipation & Volume ratio of impermeable regions \\
\hline Initial guess I & 24.0 & 0.25 \\
Optimized design I & 5.1 & 0.10 \\
\hline Initial guess II & 78.9 & 0.25 \\
Optimized design II & 5.1 & 0.10 \\
\hline Initial guess III & 36.8 & 0.11 \\
Optimized design III & 5.1 & 0.10 \\
\hline
\end{tabular}

the solid region:

$$
\begin{aligned}
\max _{\boldsymbol{\tau}}: & \Phi(\tau)=\mathbf{u}^{\mathrm{T}} \mathbf{K}_{11} \mathbf{u}, \\
\text { s.t. : } & \frac{V_{f}(\boldsymbol{\tau})}{V_{f}^{*}}-1 \leq 0, \\
: & {\left[\begin{array}{cc}
\mathbf{K}_{11}, & \mathbf{K}_{12} \\
\mathbf{K}_{21} & \mathbf{0}
\end{array}\right]\left[\begin{array}{l}
\mathbf{u} \\
\mathbf{p}
\end{array}\right]=\left[\begin{array}{c}
\mathbf{F}_{1} \\
\mathbf{0}
\end{array}\right], } \\
: & \boldsymbol{\tau}_{\text {min }} \leq \boldsymbol{\tau} \leq \boldsymbol{\tau}_{\max },
\end{aligned}
$$

where $V_{f}$ and $V_{f}^{*}$ are the current and the target volume of fluid inside the design domain, respectively, $\tau_{\min }$ and $\tau_{\max }$ are the move limits of design variables. In the current example, the upper fluid volume ratio is set as $V_{f}^{*}=0.9 V_{D}$ where $V_{D}=L^{2}$ is the total volume of the rectangular design domain. Other parameters are set as: $L=600$ and dynamic viscosity $\mu=1 \times 10^{-3}$.

Figs. 3 b-g) present three optimization cases, where (b-d) are the initial designs and (e-g) are the corresponding optimized results. The colors represent velocity magnitude. Although started with different topologies and discretizations, all three cases end up with the same geometry and equivalent performance. The data of the energy dissipation as well as the volume ratio are recorded in Table. 1 In addition, the optimized design possesses two $90^{\circ}$ angles at front and rear edges, which agree with the conclusions by Pironneau's work ${ }^{2}$. Fig. 4 depicts the optimization process for the five-obstacle initial guess, where the topological changes are clearly shown. First, the center obstacle disappears; then the remaining four merge two and two; and eventually, small mesh non-symmetries favour one obstacle instead of two and hence the design process converges to the well know global "footbal" minimum. This example demonstrates that the DSC approach is capable of generating explicit and optimized geometry through shape morphing as well as topological alteration. In addition, Fig. 5 shows the corresponding convergence curve, in which the jumps in the cost function (red solid line) are due to the topological changes. However, although different initial guesses shown above resulted in the same optimized solution, the problem is not convex and hence is generally dependent on the initial design. The optimized solution may hence be a local minimum as common for all other shape and topology optimization methods.

\section{2 | Fluid-channel design problem under Stokes and Navier-Stokes flow}

The second example demonstrates the layout of a fluid channel subject to flows of different Reynolds numbers. Fig. 6 a) illustrates the problem definition, where a fluid enters the design domain (brown color) horizontally from the inlet at the top left corner and exits from the outlet modelled as an open boundary at the bottom right corner. The extended regions (white color) at the inlet and the outlet are non-design domains. A no-slip boundary condition is assumed for all the internal boundaries. The flow at the inlet has a parabolic velocity profile with zero velocity at both ends and the maximum $u_{\max }$ in the middle. The dimensions of the domain are the same as in the previous example.

In order to obtain energy-efficient pipe designs, the same optimization problem as in the previous example that minimizes the dissipated energy s.t. a fluid volume constraint is first investigated. The upper volume limit of fluid inside the design domain is set to $V_{f}^{*}=0.27 V_{D}$, where $V_{D}=L^{2}$ and $L=120$. Starting with an initial design as shown in Figs. 6 b-e), which contains a circular impermeable region and fluid with volume $V_{f}=0.42 V_{D}$ in the design domain, four different flow velocities are considered and the corresponding optimized designs are given in Figs 6 (f-i). Case I is for Stokes flow with $\rho=0.0$ and $u_{\max }=0.5$ and the 


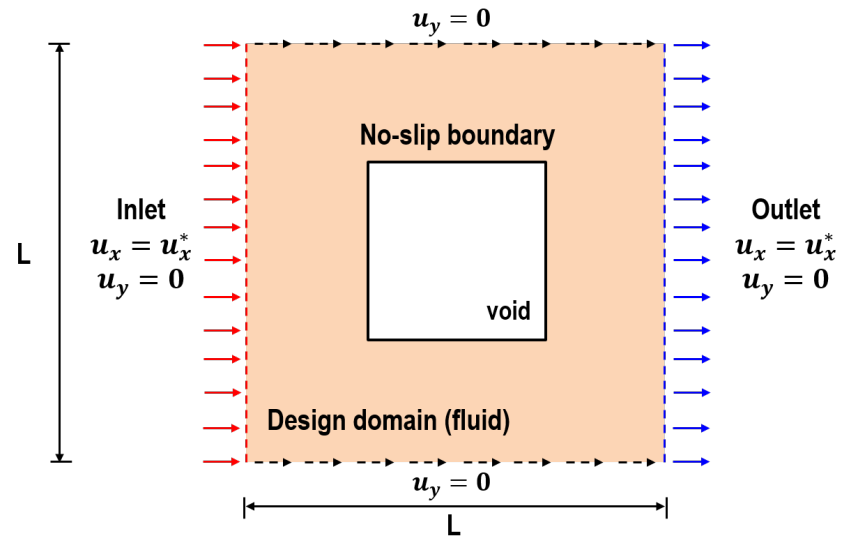

(a) Problem definition

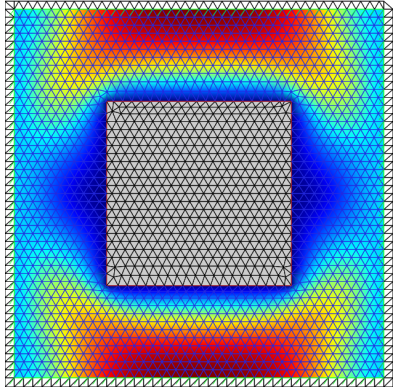

(b) Initial design I

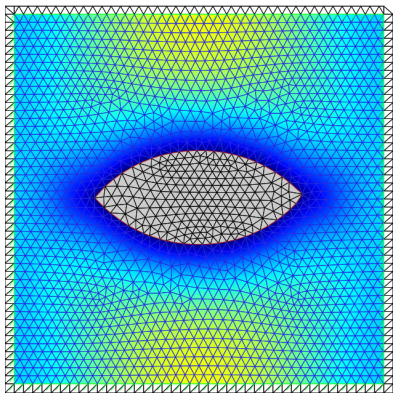

(e) Optimized design I

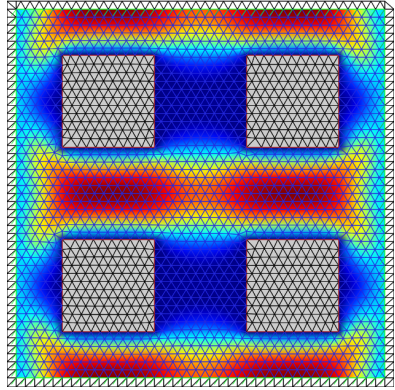

(c) Initial design II

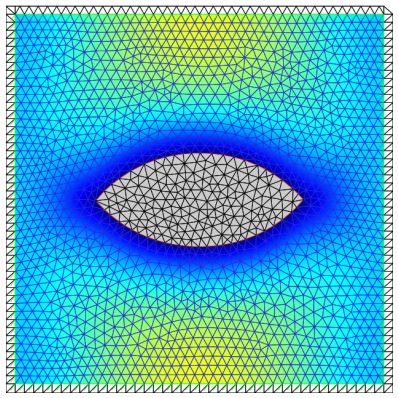

(f) Optimized design II

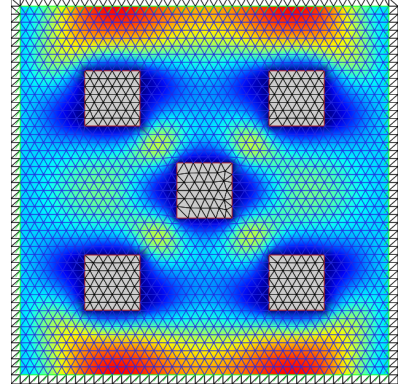

(d) Initial design III

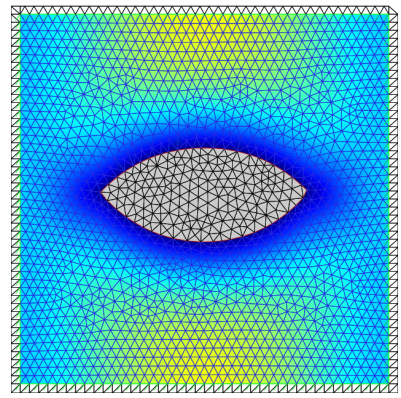

(g) Optimized design III

FIGURE 3 Shape optimization of a Stokes-flow problem: (a) problem definition (non-design domain in blue color) (b-g) each column presents the initial guess and the corresponding optimized design. The color is plotted for the magnitude of fluid velocity and the color scale is $[0,3]$

optimized design in Fig. 6 f) shows a straight channel connecting the inlet and outlet, for which the energy dissipation is 1.49. For Case II to IV, the inertial effect of the fluid are considered by assuming the same density value $\rho=1 \times 10^{-3}$ but with different flow velocities at the inlet. As the velocity is increased from (case II, $R e=60) u_{\max }=0.5$ over (case III, $\left.R e=360\right) u_{\max }=3$ to (case IV, $R e=600) u_{\max }=5$, the flow in the initial chamber bends with increasing curvature whilst the optimized geometries shown in 6 g-i) follow the same trend 3 The energy dissipation for the three designs are 1.57, 72.7 and 266 respectively.

The cross-evaluation of the optimized and the initial designs under different flow conditions are recorded in Tab. 2 Comparing the optimized Case I to IV, each pipe optimized for a particular flow condition behaves the best, i.e. has the least energy dissipation, compared to the others when evaluated at the same condition.

\footnotetext{
${ }^{3}$ The Reynolds numbers are calculated based on the reference length $L=120$, the width of the inlet and $\bar{u}=u_{\max }$.
} 


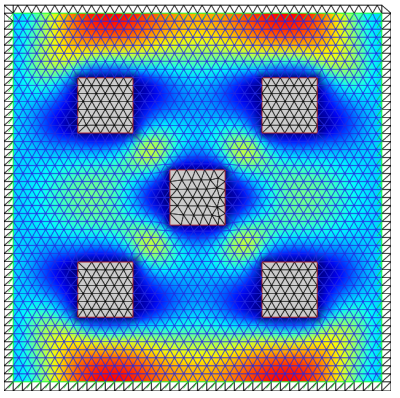

(a) Initial design

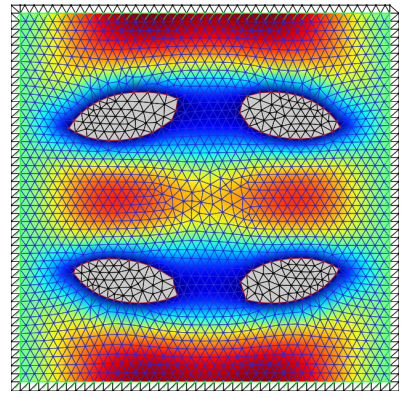

(b) Intermediate design

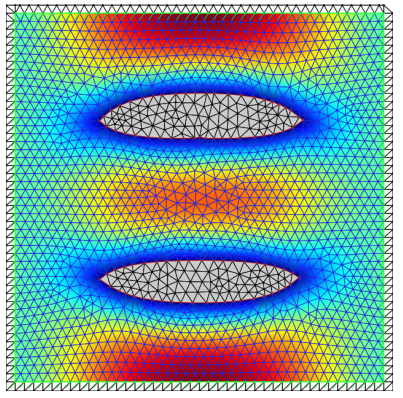

(c) Intermediate design

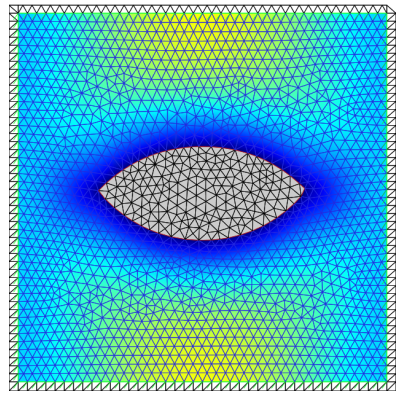

(d) Optimized design

FIGURE 4 Optimization process of a Stokes-flow problem: (a) initial guess, (b-c) intermediated design, (d) optimized design. The color is plotted for the magnitude of fluid velocity and the color scale is $[0,3]$

\section{Convergence curve}

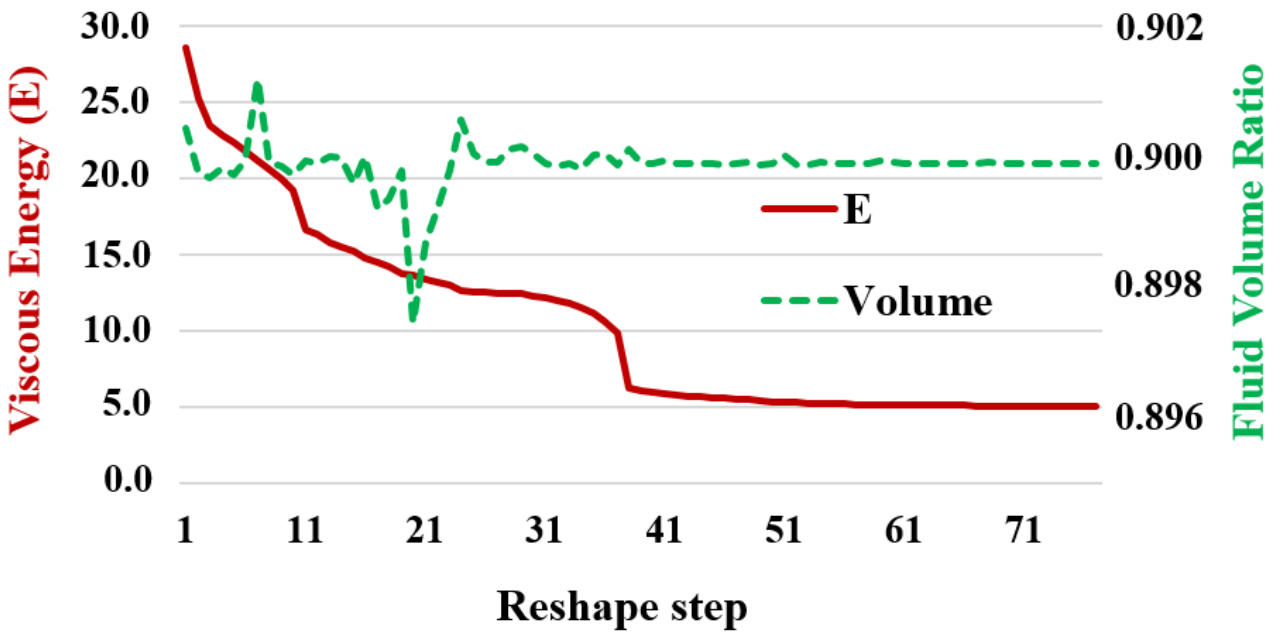

FIGURE 5 Convergence curve for the football design example with the five-hole initial guess.

The optimized results are further used as the initial guesses for minimization of energy dissipation, this time without an active volume constraints. The optimization results and data are given in Fig. 7 and Tab. 3 respectively. For the case of Stokes flow shown in Fig.7 a), the optimized chamber almost occupies the entire design domain and the volume ratio is 0.97. By increasing the velocity of the Navier-Stokes flow from $u_{\max }=0.5$ to $u_{\max }=5.0$, corresponding to the previous study, the channels shown in Figs. 7 b-d) become narrower. The performance of the optimized designs without volume constraints are better than all the designs considered in the previous study, as seen from Tab. 2 and Tab. 3

This study reveals that for high-Re flow (Case IV), it is more energy efficient to pass through a narrow channel than a wide chamber, since the former prevents undesirable and strong flow recirculation as that appearing in the latter. Oppositely, low-Re flow (Case I) can pass through a chamber with almost no recirculation. It prefers a chamber-like design with less energy loss than a narrow channel due to smaller wall-friction.

\section{3 | Fluid switch design problem}

A fluid-switch is a passive fluid control device, which is designed to divert different flows to desirable outputs by leveraging the inertia force. In this section, the proposed approach is applied to design such a device. The problem setup is inspired by an earlier work ${ }^{20}$ and is illustrated in Fig. 8 where two different flows with the same velocity but different densities are considered. The flows enter the inlet with a parabolic velocity profile, with maximum velocity 1 . The two flows have densities of $\rho_{1}=1 \times 10^{-3}$ 


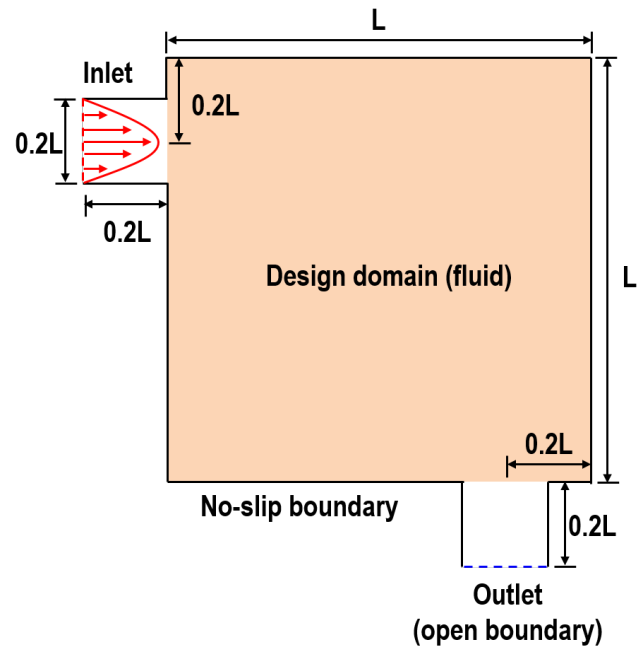

(a) Problem definition

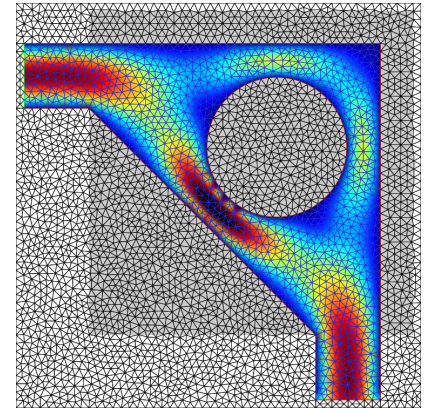

(b) Initial design I

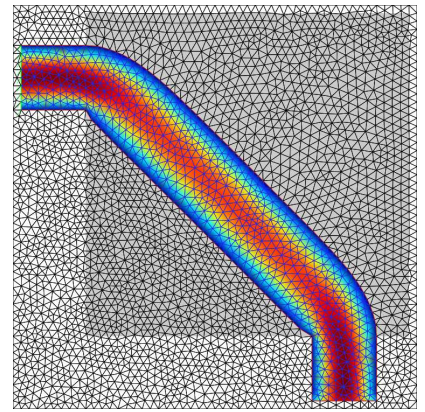

(f) Optimized design I

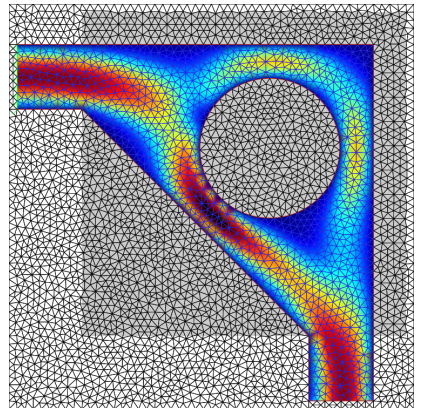

(c) Initial design II

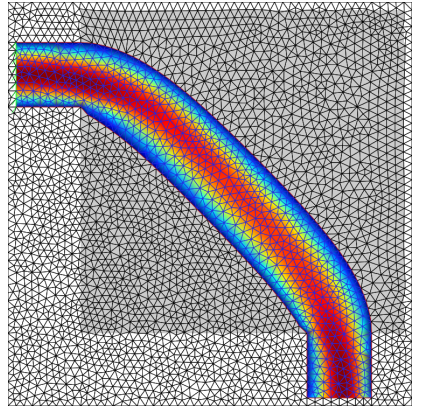

(g) Optimized design II

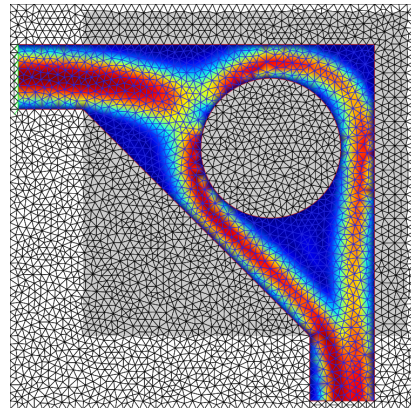

(d) Initial design III

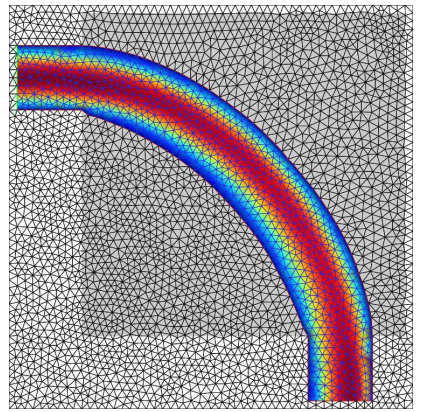

(h) Optimized design III

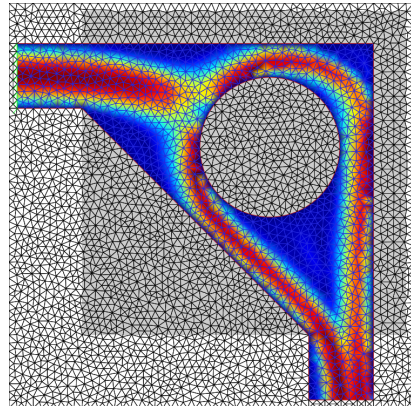

(e) Initial design IV

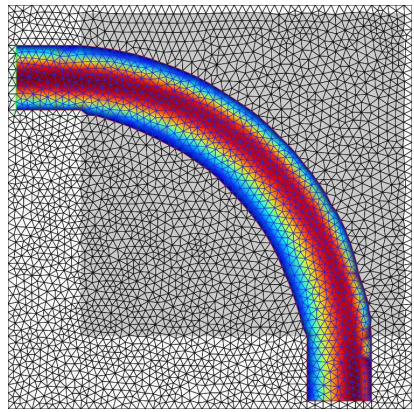

(i) Optimized design IV

FIGURE 6 Fluid-channel design problem: (a) problem definition; (b-g) (from the left column to the right) the initial guess and the corresponding optimized design for (I) Stokes flow $u_{\max }=0.5$; (II) Navier-Stokes flow $\rho=1 \times 10^{-3}, u_{\max }=0.5$; (III) Navier-Stokes flow $\rho=1 \times 10^{-3}, u_{\max }=3$; (IV) Navier-Stokes flow $\rho=1 \times 10^{-3}, u_{\max }=5$. Colors indicate fluid velocity magnitude and the color scales are (from the left column to the right) $[0,0.5],[0,0.5],[0,3.0]$ and $[0,5.0]$, respectively.

and $\rho_{2}=5 \times 10^{-5}$, respectively. They are referred to as the high- and low-Re flow, respectively in the following discussion. The design goal here is to find an optimized geometry in the design domain (in brown color), which facilitates the high- $R e$ flow exiting through outlet 2 and the low-Re flow through outlet 1. Both of the outlets are modeled as open boundary conditions. An 
TABLE 2 Cross-check for the fluid-channel problem in Fig. 6

\begin{tabular}{c|c|c|c|c}
\hline & $\begin{array}{c}\text { Viscous } \\
\text { energy } \\
\text { dissipation for } \\
\text { Stokes flow, } \\
\mathbf{u}_{\max }=0.5\end{array}$ & $\begin{array}{c}\text { Viscous } \\
\text { energy } \\
\text { dissipation } \\
\text { for Navier- } \\
\text { Stokes flow, } \\
\mathbf{u}_{\max }=0.5\end{array}$ & $\begin{array}{c}\text { Viscous } \\
\text { energy } \\
\text { dissipation } \\
\text { for Navier- } \\
\text { Stokes flow, } \\
\mathbf{u}_{\max }=3\end{array}$ & $\begin{array}{c}\text { Viscous } \\
\text { energy } \\
\text { dissipation } \\
\text { for Navier- } \\
\text { Stokes flow, } \\
\mathbf{u}_{\max }=5\end{array}$ \\
\hline Initial design & 1.4 & 1.7 & 158.5 & 804.6 \\
\hline Design I & $\mathbf{1 . 4 9}$ & 1.61 & 95.4 & 399 \\
\hline Design II & 1.51 & $\mathbf{1 . 5 7}$ & 81.3 & 331 \\
\hline Design III & 1.63 & 1.66 & $\mathbf{7 2 . 7}$ & 271 \\
\hline Design IV & 1.70 & 1.73 & 73.8 & $\mathbf{2 6 6}$ \\
\hline
\end{tabular}

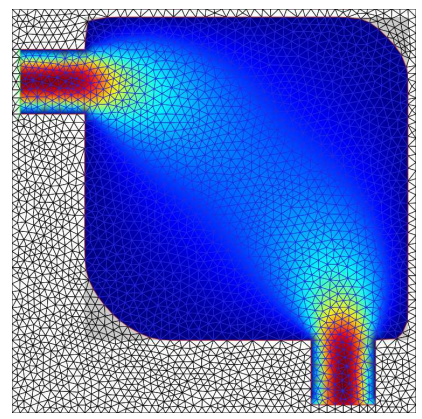

(a) Optimized design I

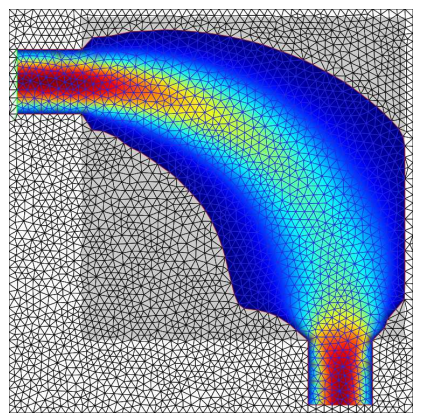

(b) Optimized design II

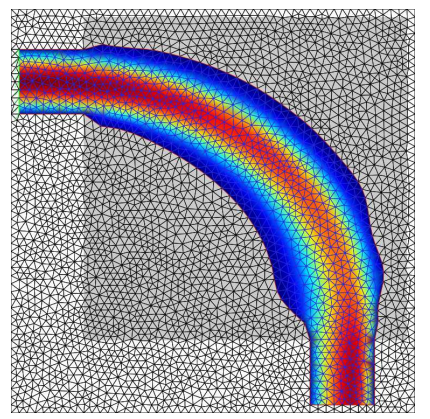

(c) Optimized design III

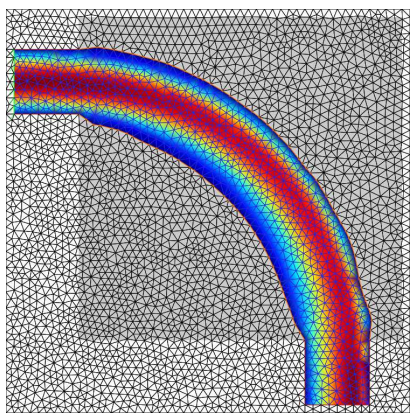

(d) Optimized design IV

FIGURE 7 Optimized designs without volume constraints: (I) Stokes flow $u_{\max }=0.5$; (II) Navier-Stokes flow $\rho=1 \times 10^{-3}$, $u_{\max }=0.5$; (III)Navier-Stokes flow $\rho=1 \times 10^{-3}, u_{\max }=3$. Colors indicate fluid velocity magnitude and the color scales are (from the left to the right) $[0,0.5],[0,0.5],[0,3.0]$ and $[0,5.0]$, respectively.

TABLE 3 Statistics of the optimized pipe design in Fig. 7 (without volume constraints)

\begin{tabular}{c|c|c|c|c}
\hline & Design I & Design II & Design III & Design IV \\
\hline $\begin{array}{c}\text { Viscous energy } \\
\text { dissipation }\end{array}$ & 0.75 & 0.96 & 62.0 & 239.7 \\
\hline $\begin{array}{c}\text { Volume ratio of fluid in } \\
\text { design domain }\end{array}$ & 0.97 & 0.68 & 0.37 & 0.30 \\
\hline
\end{tabular}

optimization problem aiming at maximum the outflow at each exit, respectively, can be formulated as:

$$
\begin{aligned}
& \max _{\tau} \text { : } \quad C(\tau)=\int_{\Gamma_{2}} \mathbf{u}_{\mathbf{h}} \cdot \mathbf{n} d \Gamma+\int_{\Gamma_{1}} \mathbf{u}_{\mathbf{l}} \cdot \mathbf{n} d \Gamma, \\
& \text { s.t. : } \quad \frac{\mathbf{u}_{\mathbf{h}}{ }^{\mathrm{T}} \mathbf{K}_{11} \mathbf{u}_{\mathbf{h}}}{\alpha \Phi_{0}}-1 \leq 0 \text {, } \\
& : \quad \frac{V(\tau)}{V^{*}}-1 \leq 0, \\
& : \quad\left[\begin{array}{cc}
\mathbf{C}\left(\mathbf{u}_{\mathbf{h}}\right)+\mathbf{K}_{11}, & \mathbf{K}_{12} \\
\mathbf{K}_{21} & \mathbf{0}
\end{array}\right]\left[\begin{array}{l}
\mathbf{u}_{\mathbf{h}} \\
\mathbf{p}_{\mathbf{h}}
\end{array}\right]=\left[\begin{array}{c}
\mathbf{F}_{\mathbf{h}} \\
\mathbf{0}
\end{array}\right], \\
& : \quad\left[\begin{array}{cc}
\mathbf{C}\left(\mathbf{u}_{\mathbf{l}}\right)+\mathbf{K}_{11}, & \mathbf{K}_{12} \\
\mathbf{K}_{21} & \mathbf{0}
\end{array}\right]\left[\begin{array}{c}
\mathbf{u}_{\mathbf{1}} \\
\mathbf{p}_{\mathbf{1}}
\end{array}\right]=\left[\begin{array}{c}
\mathbf{F}_{\mathbf{1}} \\
\mathbf{0}
\end{array}\right]
\end{aligned}
$$


where $\mathbf{u}_{\mathbf{h}}$ and $\mathbf{u}_{\mathbf{l}}$ denote the velocity of the high- and low-Re flow cases, respectively. In the above formulation, the first constraint restricts the viscous energy dissipation for the high- $R e$ flow, where $\Phi_{0}$ denotes the viscous energy dissipation of the initial guess and $\alpha$ is an application dependent ratio. The second constraint is on the volume of the fluid region, which helps generate a pipe-like design ${ }^{20}$. Other parameters are the same as the previous examples.

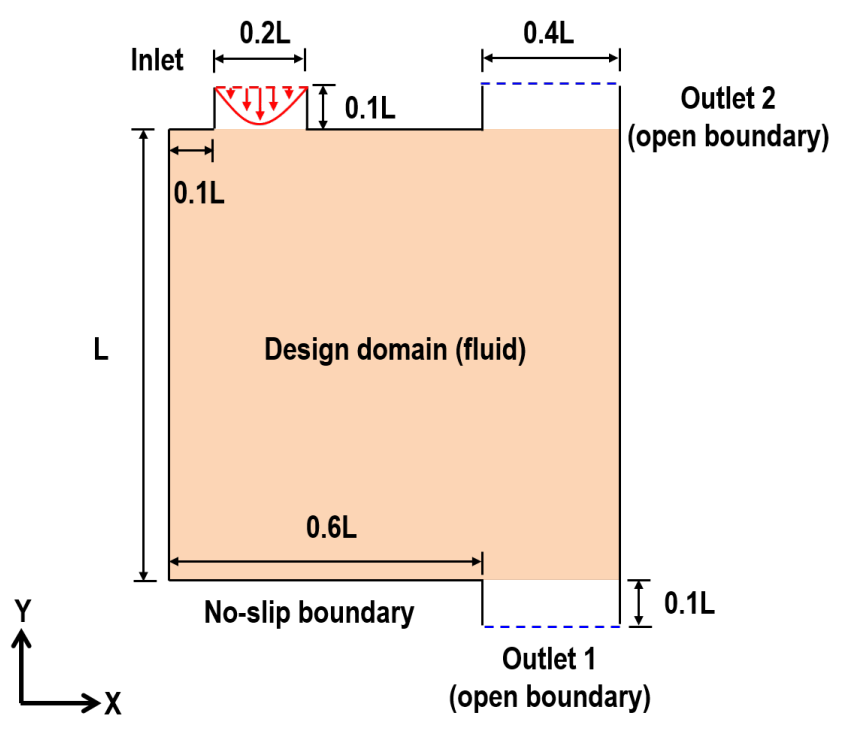

FIGURE 8 Problem definition of the fluid-switch design

Fig. 9 shows a reference fluid-switch design and the corresponding profiles of both high- and low-Re flows. The magnitude of the fluid velocity and the flow direction 4 are plotted in Figs. 9 (a-d), respectively. The outward fluid velocity profiles at each outlet for different flow cases are plotted in Fig. 9 e). For the high-Re case (Load 1), flow recirculation is observed at the outlet 1 and thus there is fluid both entering and exiting from the open boundary. The total amount of flow for each outlet is recorded in Table 4 For the reference switch, $65.4 \%$ of the high-Re flow is diverted to outlet 2 and $76.1 \%$ of low-Re flow exits from outlet 1. Before presenting the optimized designs for the fluid switch problem, a potential drawback of the DSC based shape and topology optimization framework appearing for more complex fluid design problems needs to be addressed.

\subsection{1 | Sharp features and the need for regularization}

The fluid switch design problem is first optimized based on the initial guess shown in Fig. 9 The dissipation term $\alpha$ is set to $\alpha=1.5$ and the upper volume fraction $V^{*}$ equals that of the reference design. Preliminary studies reveal a boundary instability issue as illustrated in fig. 10 where a needle-like feature appears during the optimization process. The needle guides most of the incoming fluid of the high-Re case to outlet 2 by inducing a strong recirculation near outlet 1 such that additional flow enters the domain from the open boundary outlet 1 . Hence it further contributes to the objective value which can be seen quantified in Table 4 However, the needle feature is unstable as it will gradually become thinner and thinner until it eventually is removed by a DSC mesh update due to element degeneration. Since the needle is beneficial for directing the high-Re flow to outlet 2, it will start to form again once it has been removed. Such feature loss and reappearing will keep repeating, resulting in a non-convergent optimization process.

Partly, the problem is due to a competition between the objective function and the minimum length-scale introduced by the DSC approach. However, more importantly, local flow features are not sufficiently accurately captured around a needlelike feature described by one finite element. Hence, as described so far, the DSC approach is unable to prevent mesh-related,

\footnotetext{
${ }^{4}$ The length of the arrows is not proportional to the magnitude of the velocity.
} 
erroneous features to appear in more complex design situations. To remedy this shortcoming and to make the DSC fluid design method applicable to general flow problems, the following regularization scheme is proposed.

\subsection{2 | Boundary smoothing}

The use of filtering in shape optimization is known as an effective way to obtain meaningful results with controllable smoothness of the optimized geometries. It helps avoiding numerical deficiencies, such as jagged boundaries due to the approximation errors of the FE analysis. For the FE based shape optimization, where the exterior nodes of a computational grid are designable, one can employ consistent or inconsistent filtering schemes known from the literature. The former corresponds to algorithms where the design sensitivities are obtained by differentiating the design responses w.r.t. actual design variables via the chain rule. The latter refers to heuristic "sensitivity filtering" approaches, for which a smoothed version of the consistent sensitivity values are leveraged for shape deformation. Implementation of consistent filtering usually utilizes two separate meshes as in ${ }^{13}$, where the nodes of a parent mesh are the design variables and a child mesh represents the actual geometry. The latter is based on filtering the nodal position of the parent mesh. As a result, the actual geometry has a smoothed boundary and a consistent sensitivity analysis can be carried out. Another approach is by using a convex-hull based parameterization as implemented in ${ }^{[12}$, where a consistent sensitivity w.r.t. the designable control points is derived via the chain rule. Apart from the consistent scheme, it is possible to only restrict the nodal update as an average of that of its neighbors 40 . However, it may lead to an inconsistent optimization and the optimality can hardly be verified. With the DSC method, considering the fact that the topology (element connectivity) of the mesh is continuously changing during the optimization process by local mesh adaption, it is impractical to have two separate meshes as in $\frac{113}{13}$ or have an extra parameterization for the boundary nodes as in $\frac{12}{12}$, because inversely calculating a parent mesh or a parametrization is non-trivial whenever the mesh changes. Hence, a new regularization scheme is proposed as follows, which operates only on one set of boundary nodes.

The new regularization scheme is based on applying a filtering matrix $\mathbf{M}$ over the original DSC updating scheme Eq. (1). The new node updating scheme is given as:

$$
\tilde{\mathbf{w}}^{\text {new }}=\mathbf{M} \cdot \mathbf{w}^{\text {new }}=\mathbf{M} \cdot\left(\tilde{\mathbf{w}}^{\text {old }}+\Delta \tilde{\mathbf{w}}\right),
$$

where

$$
\Delta \tilde{\mathbf{w}}_{i}=\tau_{i} \mathbf{n}_{i}
$$

For node $i$, each item of $\mathbf{M}_{i j}(j=1: n)$ in the filter matrix represents the filtering impact from its neighbor node $j$ whose value is defined according to its chord distance along the interface:

$$
\mathbf{M}_{i j}=\frac{\exp \left(-\operatorname{dist}\left(\mathbf{x}_{j}-\mathbf{x}_{i}\right)^{2} / r^{2}\right)}{\sum_{j \in \mathbf{N}_{i}} \exp \left(-\operatorname{dist}\left(\mathbf{x}_{j}-\mathbf{x}_{i}\right)^{2} / r^{2}\right)},
$$

where $r$ is the radius of the filter and the set $\mathbf{N}_{i}=\left\{\mathbf{x} \mid \operatorname{dist}\left(\mathbf{x}-\mathbf{x}_{i}\right)<r\right\}$ covers the region of interest for smoothing. Essentially, this scheme filters the nodal positions of a mesh rather than the nodal update, as shown in Fig. 11 . It has the same smoothing effect as the "shape filtering" proposed by $\underline{13}$, but differently, it operates only on one boundary curve which serves both as the design variable and representing the actual boundary.

With this filtering scheme, a consistent sensitivity analysis for a general functional $f\left(\tilde{\mathbf{w}}^{\text {new }}\right)$ w.r.t. the design variable $\tau_{i}$ is given as:

$$
\left.\frac{d f\left(\tilde{\mathbf{w}}^{\text {new }}\right)}{d \tau_{i}}\right|_{\tau=0}=\left.\frac{d f\left(\mathbf{M} \cdot \tilde{\mathbf{w}}^{\text {old }}+\mathbf{M} \cdot \Delta \tilde{\mathbf{w}}\right)}{d \Delta \tilde{\mathbf{w}}_{i}}\right|_{\tau=0} \cdot \frac{d \Delta \tilde{\mathbf{w}}_{i}}{d \tau_{i}}=\left.\frac{d f\left(\mathbf{M} \cdot \tilde{\mathbf{w}}^{\text {old }}+\mathbf{M} \cdot \Delta \tilde{\mathbf{w}}\right)}{d \Delta \tilde{\mathbf{w}}_{i}}\right|_{\tau=0} \cdot \mathbf{n}_{i} \cdot
$$

The above equation implies that in order to perform sensitivity analysis for the regularized scheme in each design iteration, an intermediate shape defined by $\mathbf{w}^{*}=\mathbf{M} \cdot \tilde{\mathbf{w}}^{\text {old }}$ (for $\tau=0$ ) must be first formed and the associated design responses need to be evaluated. Invoking additional function evaluation at this intermediate shape is a drawback of the proposed scheme due to the extra computational cost. However, the proposed regularization scheme turns out to be suitable for DSC-based shape optimization and it is effective in yielding converged designs for more complex flow problems.

\subsection{3 | Optimization results with regularization}

With the proposed regularization scheme, the fluid-switch optimization problem is solved with a dissipation term $\alpha=1.5$ while the upper volume fraction $V^{*}$ is equal to that of the reference design. The optimized switch design I is shown in Fig. 12 which 
TABLE 4 Percentage of net flow exiting each outlet for different fluid switch designs

\begin{tabular}{c|c|c|c|c}
\hline & $\begin{array}{c}\text { High-Re, } \\
\text { outlet 1 }\end{array}$ & $\begin{array}{c}\text { High-Re, } \\
\text { outlet 2 }\end{array}$ & $\begin{array}{c}\text { Low-Re, } \\
\text { outlet 1 }\end{array}$ & $\begin{array}{c}\text { Low-Re, } \\
\text { outlet 2 }\end{array}$ \\
\hline Initial guess & $34.6 \%$ & $65.4 \%$ & $76.1 \%$ & $23.9 \%$ \\
\hline $\begin{array}{c}\text { Switch design with } \\
\text { sharp feature }\end{array}$ & $0.0 \%$ & $100 \%$ & $89.0 \%$ & $11.0 \%$ \\
\hline Switch design I & $2.0 \%$ & $98.0 \%$ & $92.7 \%$ & $7.3 \%$ \\
\hline Switch design II & $7.3 \%$ & $92.7 \%$ & $91.0 \%$ & $9.0 \%$ \\
\hline
\end{tabular}

exhibits a smooth boundary and a better performance than the reference design. Nearly $98.0 \%$ of the high-Re flow and $92.7 \%$ of low-Re flow are diverted to the desirable outlets, respectively. If the constraint on the energy dissipation is tightened to $\alpha=1.0$, the second optimization result switch II shown in Fig. 13 comprises an enlarged channel. As a result, 92.7\% of the high-Re flow and $91.0 \%$ of low-Re flow are guided to the desirable outlets, which is slightly worse than that of the design I. No boundary instability issues are observed in these two optimizations with the added regularization.

For the fluid switch design example, constraining the viscous dissipation energy turns out to be an indirect but effective approach to control the length scale for the fluid channel design. Without such a restriction employed, a extremely narrow channel can appear to guide more flow to outlet 2 for the high-Re load and thus further improve the objective value. However, it may cause computational difficulties since the fluid velocity and the local Reynolds number increase when the channel shrinks. Therefore, properly constraining the dissipation energy is important to ensure a meaningful channel design.

\section{7 | CONCLUSIONS}

In this work, numerical shape optimization of 2D fluid-flow problems is advanced by employing the DSC method. Particularly, the developed methodology allows shape optimization combined with topological changes. Optimized geometries are obtained for problems of low to moderate Reynolds numbers. The solution offers great flexibility for flow channel design and related applications for shape optimization, as it can handle large boundary deformations efficiently. Particularly, the geometry in an explicit format can facilitate subsequent CAD transfer to parametric models. For simple energy dissipation problems, obtained solutions appear to be stable without additional regularization. For more complex objective functions, a new regularization scheme is effective in suppressing sharp features and ensuring converged designs, at the cost of the need to introduce an intermediate geometry to be formed and an additional finite element analysis. Essentially, the boundary smoothing proposed in this paper regularizes the optimization problem and generally helps the DSC based shape optimization process converge in a more stable way than the original solution. It is hence generally advised to implement the regularization in order to alleviate oscillations in the convergence and improve it if the optimization problem is sensitive to boundary changes. Indeed, a lot of work has been done for shape optimization of fluids in the past and new on-going efforts are devoted to xFEM and cutFEM methods that try to avoid remeshing. The approach presented here shows that local mesh adaption can be effective in obtaining clear and optimized shape of fluids. Future work will consider the extension to 3D. Although surely non-trivial, the extension seems straight forward and can be built on already developed 3D code for the elasticity problem 16 .

\section{ACKNOWLEDGEMENT}

The authors acknowledge the support from National Natural Science Foundation of China (Grant No. 51705311) and the support from the project "Sapere Aude TOpTEN" (Topology Optimization of Thermal ENergy systems) from the Danish Council for Independent Research (Grant No. DFF-4005-00320). Most of this work was performed when the first two authors worked at the Dept. of Mechanical Engineering of the Technical University of Denmark. 


\section{References}

1. Lighthill MJ. A new method of two dimensional aerodynamic design. Report and Memoranda. 1945;No. 2112.

2. Pironneau O. On optimum design in fluid mechanics. Journal of Fluid Mechanics. 1974;64(1):97-110.

3. Jameson A, Martinelli L, Pierce NA. Optimum aerodynamic design using the Navier-Stokes equations. Theoretical and Computational Fluid Dynamics. 1998;10(1):213-237.

4. Mohammadi B, Pironneau O. Applied Shape Optimization for Fluids. 2001.

5. Mohammadi B, Pironneau O. Shape optimization in fluid mechanics. Annual Review of Fluid Mechanics. 2004;36:255-279.

6. Katamine E, Azegami H, Tsubata T, Itoh S. Solution to shape optimization problems of viscous flow fields. International Journal of Computational Fluid Dynamics. 2005;19(1):45-51.

7. Lehnhäuser T, Schäfer M. A numerical approach for shape optimization of fluid flow domains. Computer Methods in Applied Mechanics and Engineering. 2005;194:5221-5241.

8. Braibant V, Fleury C. Shape optimal design using B-splines. Computer Methods in Applied Mechanics and Engineering. 1984;44(3):247-267.

9. Bletzinger KU, Kimmich S, Ramm E. Efficient modeling in shape optimal design. Computing Systems in Engineering. 1991;2(3):483-495.

10. Lian H, Kerfriden P, Bordas SPA. Implementation of regularized isogeometric boundary element methods for gradientbased shape optimization in two-dimensional linear elasticity. International Journal for Numerical Methods in Engineering. 2016;106(12):972-1017.

11. Lian H, Kerfriden P, Bordas SPA. Shape optimization directly from CAD: an isogeometric boundary element approach using T-splines. Computer Methods in Applied Mechanics and Engineering. 2017;317:1-41.

12. Bletzinger KU. A consistent frame for sensitivity filtering and the vertex assigned morphing of optimal shape. Structural and Multidisciplinary Optimization. 2014;49(6):873-895.

13. Le C, Bruns T, Tortorelli D. A gradient-based, parameter-free approach to shape optimization. Computer Methods in Applied Mechanics and Engineering. 2011;200:985-996.

14. Misztal MK, Bærentzen JA. Topology-adaptive interface tracking using the deformable simplicial complex. ACM Transactions on Graphics (TOG). 2012;31(3):No. 24.

15. Christiansen AN, Nobel-Jørgensen M, Aage N, Sigmund O, Bærentzen J Andreas. Topology optimization using an explicit interface representation. Structural and Multidisciplinary Optimization. 2014;49:387-399.

16. Christiansen AN, Bærentzen JA, Nobel-Jørgensen M, Aage N, Sigmund O. Combined shape and topology optimization of 3D structures. Computers \& Graphics. 2015;46:25-35.

17. Lian H, Christiansen AN, Tortorelli DA, Aage N, Sigmund O. Combined Shape and Topology Optimization for Minimization of Maximal von Mises Stress. Structural and Multidisciplinary Optimization. 2017;55(5):1541-1557.

18. Zhou M, Sigmund O. On fully stressed design and p-norm measures in structural optimization. Structural and Multidisciplinary Optimization. 2017;56(3):731-736.

19. Borrvall T, Petersson J. Topology optimization of fluids in Stokes flow. International Journal for Numerical Methods in Fluids. 2003;41(1):77-107.

20. Gersborg-Hansen A, Sigmund O, Haber RB. Topology optimization of channel flow problems. Structural and Multidisciplinary Optimization. 2005;30(3):181-192. 
21. Guest JK, Prèvost JH. Topology optimization of creeping fluid flows using a Darcy-Stokes finite element. International Journal for Numerical Methods in Fluids. 2005;66(3):461-484.

22. Aage N, Poulsen TH, Gersborg-Hansen A, Sigmund O.. Topology optimization of large scale stokes flow problems. Structural and Multidisciplinary Optimization. 2008;35(2):175-180.

23. Yoon GH. Topology optimization for stationary fluid-structure interaction problems using a new monolithic formulation. International Journal for Numerical Methods in Engineering. 2010;82(5):591-616.

24. Alexandersen J, Aage N, Andreasen C Schousboe, Sigmund O. Topology optimisation of natural convection problems. International Journal for Numerical Methods in Fluids. 2015;76(10):699-721.

25. Jenkins N, Maute K. Level set topology optimization of stationary fluid-structure interaction problems. Structural and Multidisciplinary Optimization. 2015;52:179-195.

26. Osher S, Fedkiw R. Level Set Methods and Dynamic Implicit Surfaces. 2003.

27. Zhou S, Li Q. A variational level set method for the topology optimization of steady-state Navier-Stokes flow. Journal of Computational Physics. 2008;227(4):10178-10195.

28. Duan X, Ma Y, Zhang R. Shape-topology optimization for Navier-Stokes problem using variational level set method. Journal of Computational and Applied Mathematics. 2008;222(2):487-499.

29. Challis VJ, Guest JK. Level set topology optimization of fluids in Stokes flow. International Journal for Numerical Methods in Engineering. 2009;79:1284-1308.

30. Pingen G, Waidmann M, Evgrafov A, Maute K. A parametric level-set approach for topology optimization of flow domain. Journal of Computational and Applied Mathematics. 2010;41(1):117-131.

31. Villanuevaa CH, Maute K. CutFEM topology optimization of 3D laminar incompressible flow problems. Computer Methods in Applied Mechanics and Engineering. 2017;320:444-473.

32. Zhou M, Wang MY, Li L. Structural Optimization Using Adaptive Level Set Method. Proceedings of the ASME/ISCIE 2012 International Symposium on Flexible Automation. 2012;ISFA2012-7110:1-7.

33. Allaire G, Dapogny C, Frey P. Shape optimization with a level set based mesh evolution method. Computer Methods in Applied Mechanics and Engineering. 2014;282(1):22-53.

34. Yamasaki S, Kawamoto A, Nomura T, Fujita K. A consistent grayscale-free topology optimization method using the level-set method and zero-level boundary tracking mesh. International Journal for Numerical Methods in Engineering. 2015;101:744-773.

35. Misztal MK, Erleben K, Bargteil A, et al. Multiphase flow of immiscible fluids on unstructured moving meshes. IEEE Transactions on Visualization and Computer Graphics. 2014;20(1):4-16.

36. Girault V, Raviart PA. Finite Element Approximation of the Navier-Stokes Equations. Springer-Verlag Berlin Heidelberg. 1979;.

37. Reddy JN, Gartling DK. The Finite Element Method in Heat Transfer and Fluid Dynamics. 2010.

38. Svanberg K. The method of moving asymptotes - a new method for structural optimization. International Journal for Numerical Methods in Engineering. 1987;24:359-397.

39. Pironneau O. On optimum profiles in Stokes flow. Journal of Fluid Mechanics. 1973;59(1):117-128.

40. Pironneau O. Optimal Shape Design for Elliptic Systems. 1984. 


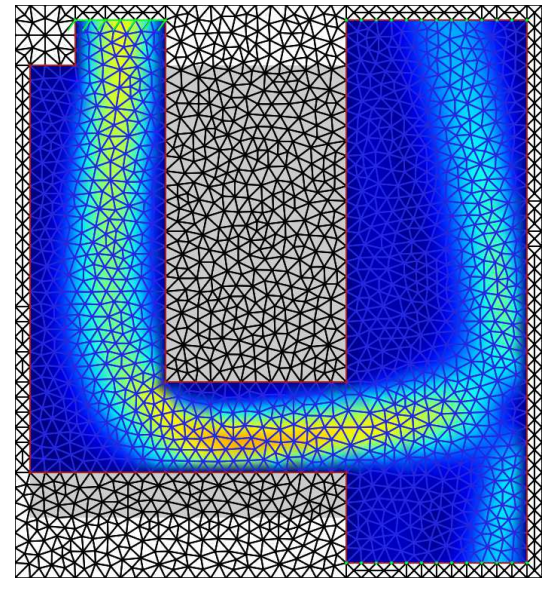

(a) High $R e$ flow, velocity magnitude

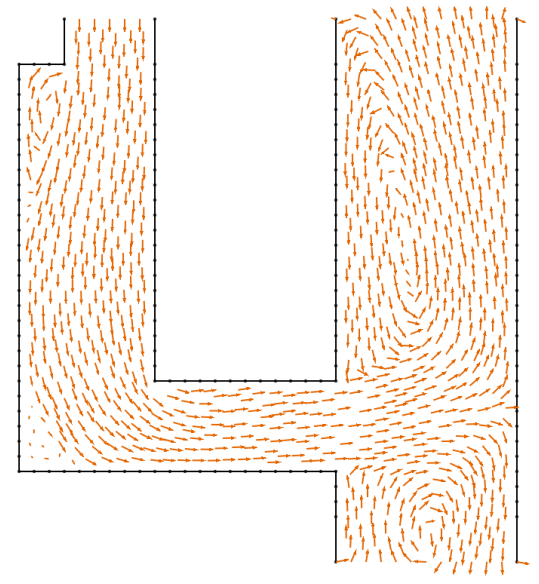

(c) High $R e$ flow, velocity direction

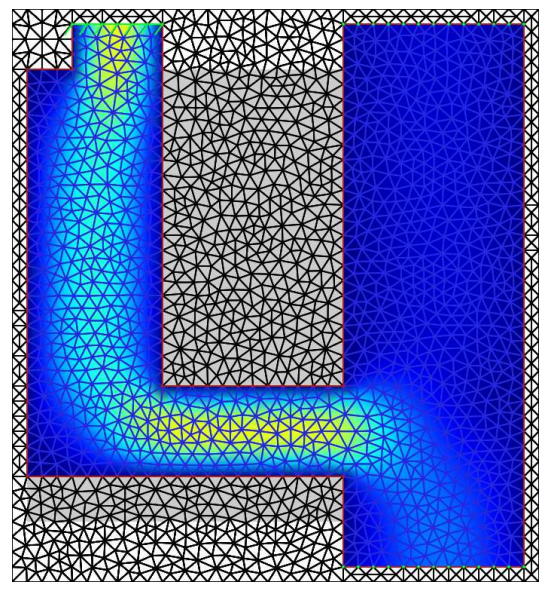

(b) Low $R e$ flow, velocity magnitude

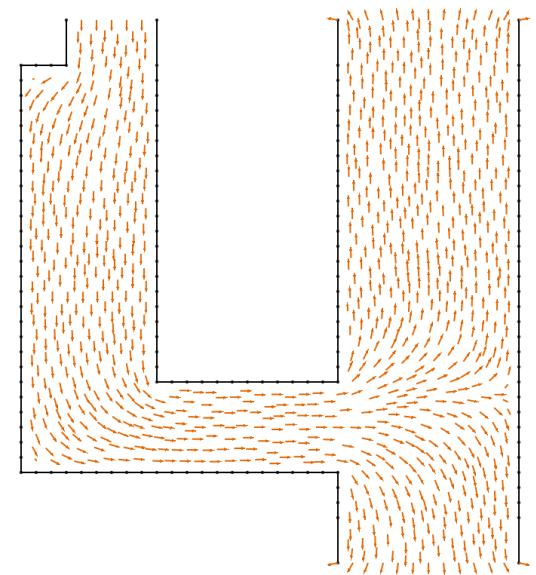

(d) Low $R e$ flow, velocity direction

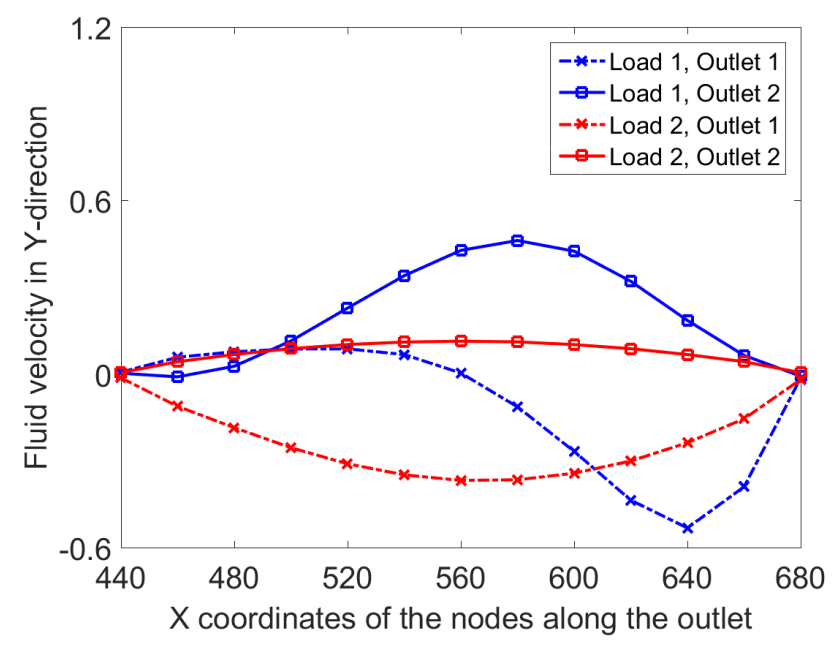

(e) Velocity profile (Y-direction) at outlets

FIGURE 9 Initial switch design: (a,c) high $R e$ flow; (b,d) high $R e$ flow; (e) fluid velocity in Y-direction at the outlets (Load 1, high $R e$ flow; Load 2, low Re flow). The color scale for the magnitude of fluid velocity is $[0.0,1.63]$. 


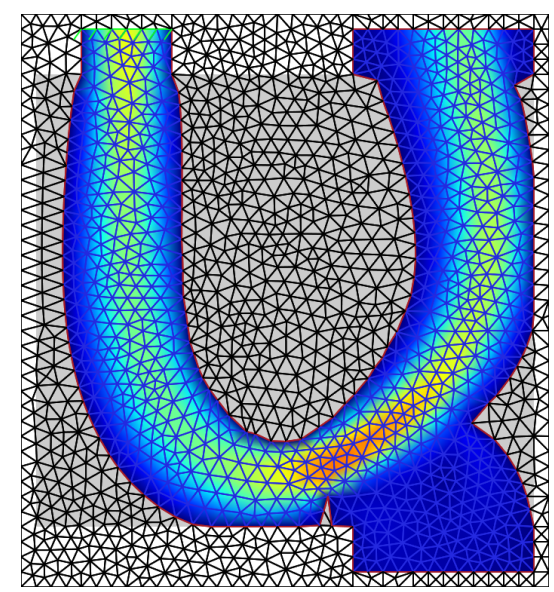

(a) High $R e$ flow, velocity magnitude

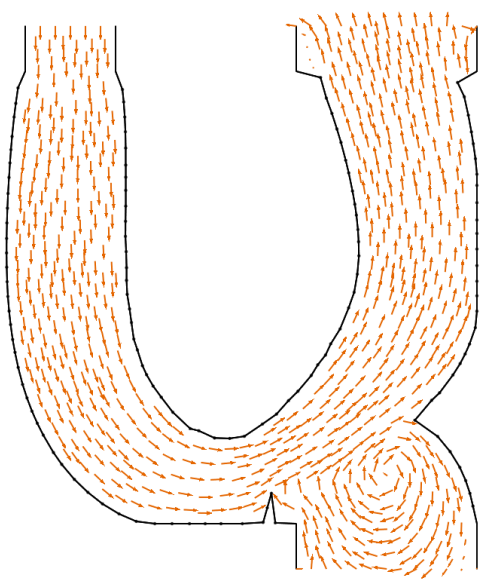

(b) High Re flow, velocity direction

FIGURE 10 An intermediate switch design without using regularization: (a) flow velocity; (b) flow direction. The color scale for the magnitude of fluid velocity is the same as that in Fig. 9

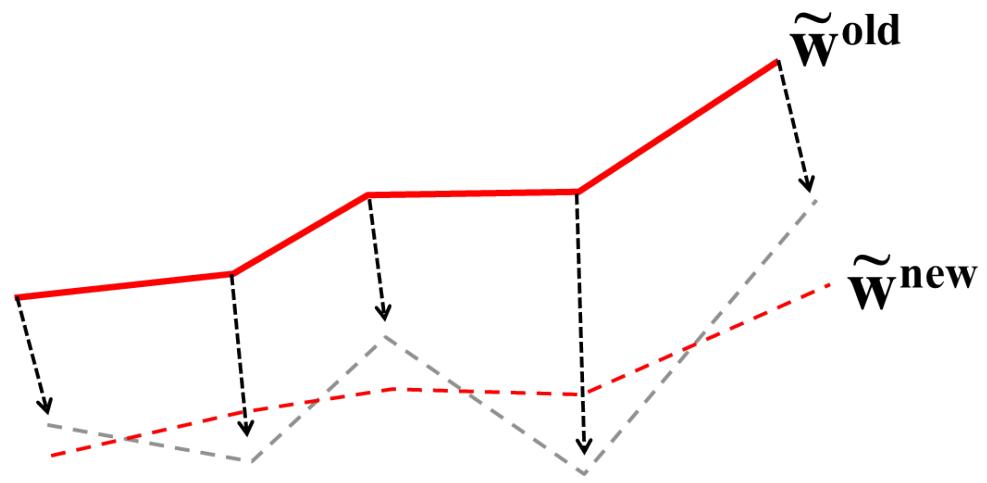

FIGURE 11 DSC update with regularization: the final boundary $\tilde{\mathbf{w}}^{\text {new }}$ is smoothed after each update. 


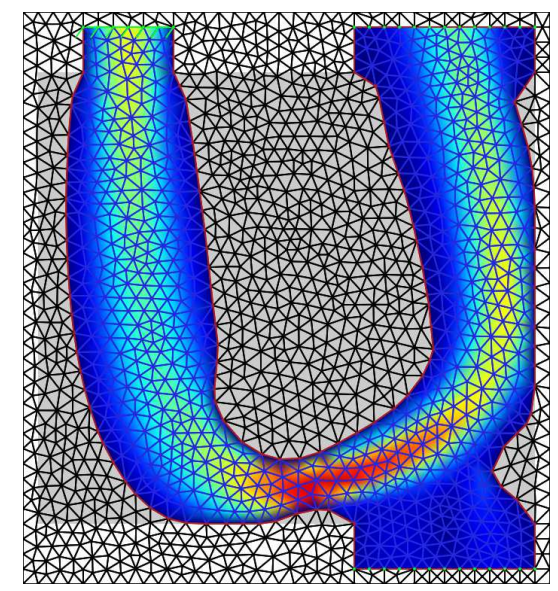

(a) High Re flow, velocity magnitude

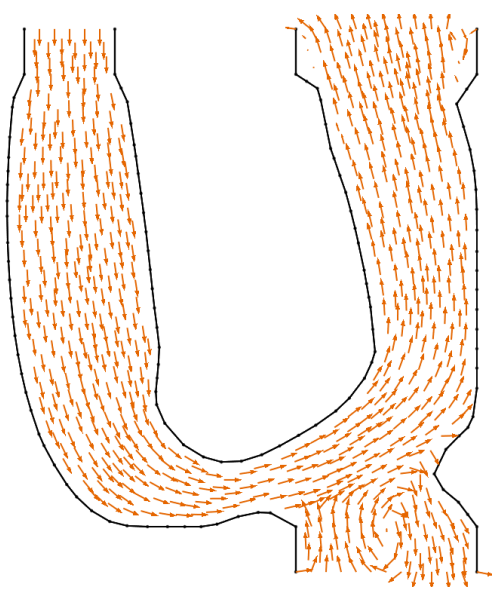

(c) High Re flow, velocity direction

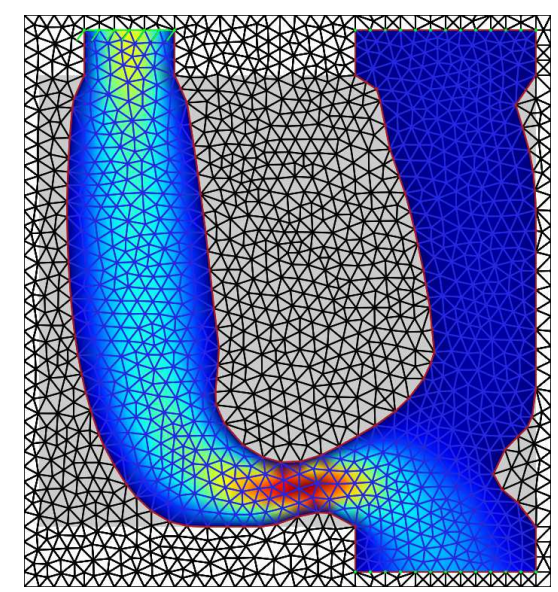

(b) Low Re flow, velocity magnitude

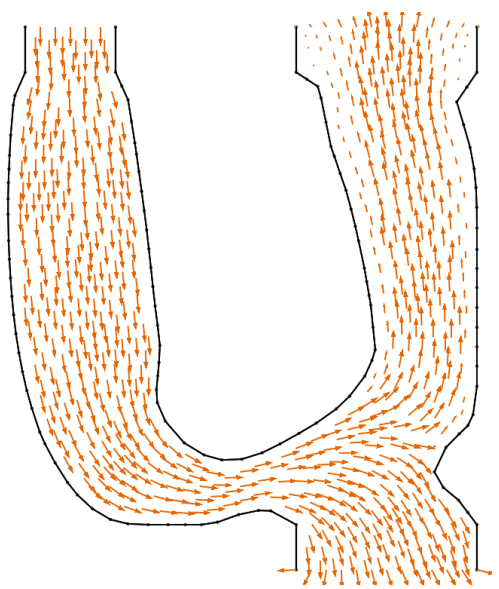

(d) Low Re flow, velocity direction

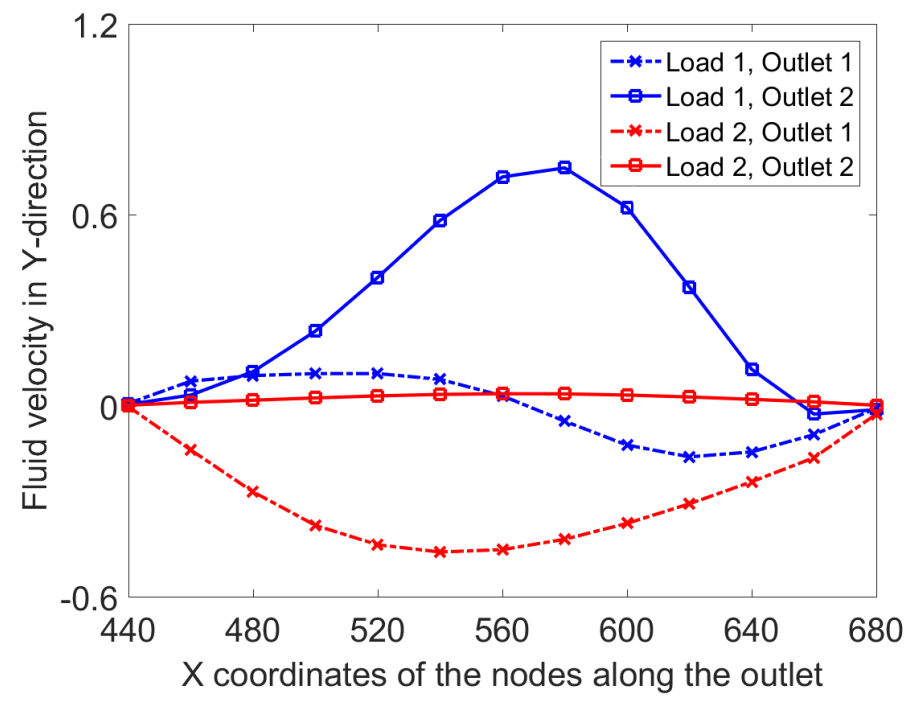

(e) Velocity profile at outlets

FIGURE 12 Optimized switch design I with a relaxed constraint $(\alpha=1.5)$ on the flow dissipation under high the $R e$ flow: (a,c) high $R e$ flow; (b,d) high $R e$ flow; (e) fluid velocity in Y-direction at the outlets (Load 1, high $R e$ flow; Load 2, low $R e$ flow). The color scale for the magnitude of fluid velocity is the same as that in Fig. 9 . 


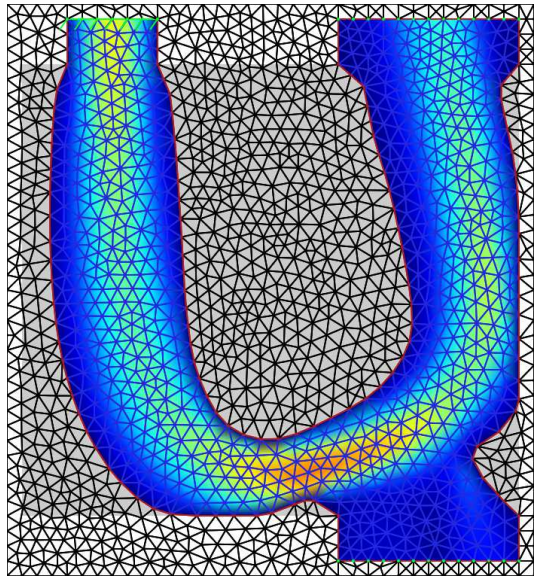

(a) High $R e$ flow, velocity magnitude

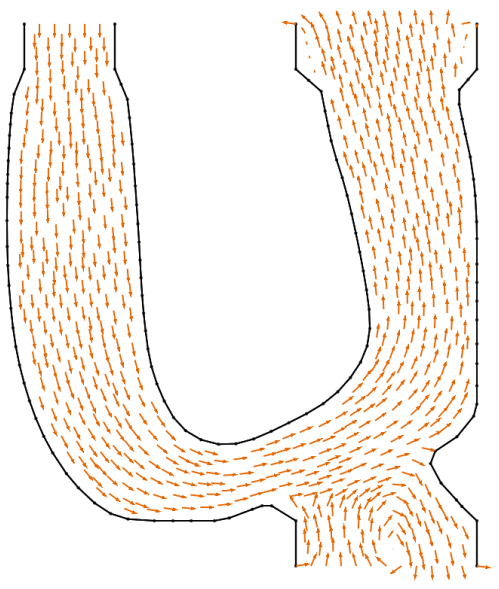

(c) High $R e$ flow, velocity direction

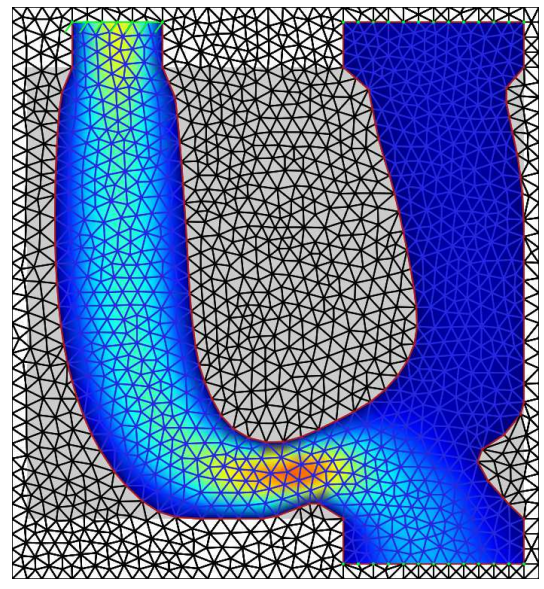

(b) Low $R e$ flow, velocity magnitude

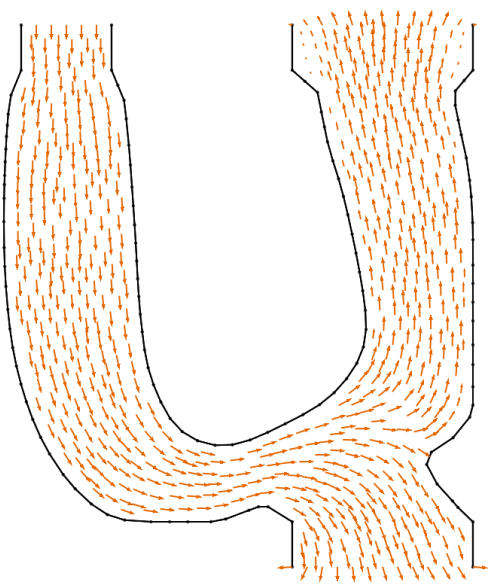

(d) Low Re flow, velocity direction

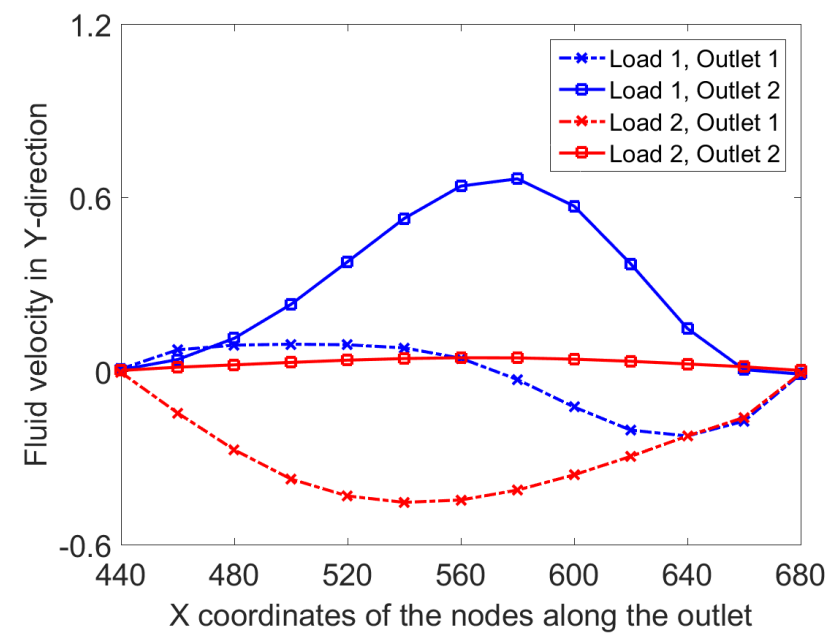

(e) Velocity (Y-direction) profile at outlets

FIGURE 13 Optimized switch design II with a tight constraint $(\alpha=1.0)$ on the flow dissipation under high the $R e$ flow: (a,c) high $R e$ flow; (b,d) high $R e$ flow; (e) fluid velocity in Y-direction at the outlets (Load 1, high $R e$ flow; Load 2, low $R e$ flow). The color scale for the magnitude of fluid velocity is the same as that in Fig. 9 
$\square$ 